\title{
IFU observations of the GRB 980425/SN 1998bw host galaxy: emission line ratios in GRB regions ${ }^{\star}$
}

\author{
L. Christensen ${ }^{1}$, P. M. Vreeswijk ${ }^{2}$, J. Sollerman ${ }^{2,3}$, C. C. Thöne ${ }^{2}$, E. Le Floc'h ${ }^{4}$, and K. Wiersema ${ }^{5}$ \\ 1 European Southern Observatory, Casilla 19001, Santiago 19, Chile \\ e-mail: lichrist@eso.org \\ 2 Dark Cosmology Centre, Niels Bohr Institute, University of Copenhagen, Juliane Maries Vej 30, 2100 Ø, Denmark \\ 3 Department of Astronomy, AlbaNova, 10691 Stockholm University, Sweden \\ 4 Spitzer Fellow, Institute for Astronomy, 2680 Woodlawn Drive, Honolulu HI 96822, USA \\ 5 University of Leicester, University Road, Leicester, LE1 7RH, UK
}

Received 3 April 2008 / Accepted 16 July 2008

ABSTRACT

\begin{abstract}
Context. The collapsar model predicts that the progenitors of Gamma-ray Bursts (GRBs) are metal poor in Fe group elements. The existence of low metallicity stellar populations could manifest itself in the characteristics of the GRB site immediate environment in the host galaxy.

Aims. We analyse the strong emission lines from the sub-luminous host galaxy of GRB 980425, which showed the first connection with a supernova explosion (SN 1998bw). The host is of sufficient size to allow detailed resolved spectroscopy of individual H II regions and to search for regions with peculiar properties close to the the GRB site.

Methods. Using integral field spectroscopy with VIMOS we study most of the high surface brightness part of the host including the $\mathrm{H}$ II region where the supernova and GRB occurred.

Results. The star formation rate, reddening, equivalent width and stellar mass in the GRB region is similar to other $\mathrm{H}$ II regions in the host. Extreme values arise in the only region that shows emission lines from Wolf-Rayet stars, a region that is located $800 \mathrm{pc}$ in projection from the GRB site. Strong emission line diagnostics of all H II regions imply oxygen abundances between 0.3 and 0.8 solar with the lowest values arising in the WR and GRB regions. Including uncertainties from the metallicity diagnostics, all metallicities are similar to within $3 \sigma$. We demonstrate that there is a good agreement between the luminosity weighted and mass weighted specific star formation rates (SSFR) in individual young H II regions. While the global average of the SSFR is similar to high redshift GRB hosts, there are significant variations between individual resolved H II regions. Comparing the measured emission line ratios of low redshift GRB hosts to theoretical models and observations of field galaxies, we find that GRBs are present in different environment metallicities while the regions of their origin are consistently very young. Similar line ratios of GRB hosts compared with those of the WR region can arise in spatially unresolved galaxies with bright H II regions close to the GRB location.
\end{abstract}

Key words. gamma rays: bursts - galaxies: abundances - galaxies: individual: GRB 980425

\section{Introduction}

The association of the Gamma-ray Burst GRB 980425 with the supernova SN 1998bw (Galama et al. 1998) was initially questioned because of the low redshift of the supernova host galaxy ESO 184-G82, $z=0.0085$. The corresponding isotropic energy output of GRB 980425 was low $\left(E \sim 10^{49}\right.$ erg) compared with other GRBs known at that time (at $z \gtrsim 1$ with $E \sim 10^{52} \mathrm{erg}$ ). Since then, more apparently under-luminous, low redshift GRB events have been detected. The definite proof of a supernova connection originated with the discovery of SN type Ic signatures in the afterglow spectra of a couple of long duration GRBs (Hjorth et al. 2003; Stanek et al. 2003; Malesani et al. 2004). While the energetics of GRB 980425 suggested an underluminous event, there was no indication that its accompanying supernova was peculiar for a GRB-SN (Kaneko et al. 2007).

About 500 GRBs have been observed and optical afterglows have been detected for about a third ${ }^{1}$. While bursts detected by

\footnotetext{
* Based on observations collected at the European Southern Observatory, Chile: programme ID 077.D-0488(A), and archive programmes: 64.H-0375(A), 66.D-0576(A) and 275.D-5039(A).

${ }_{1}$ http://www .mpe.mpg.de/ jcg/grbgen.html
}

the BeppoSAX satellite typically had redshifts $z \sim 1$, those detected by the Swift satellite (Gehrels et al. 2004) have an average redshift of 2.2 (Jakobsson et al. 2006) $^{2}$. The small angular size of the high redshift host galaxies (typically less than a few arcseconds) implies that a closer examination of the nature of the hosts requires integrated spectra or broad band images for the faintest hosts. Images of GRB hosts indicate that GRBs are located preferentially in sub-luminous (Le Floc'h et al. 2003) actively star-forming, young galaxies (Christensen et al. 2004a). The distribution of GRB afterglow locations within their hosts follow in general the bright continuum light distribution of the host galaxies (Bloom et al. 2002; Fruchter et al. 2006).

Models of single star collapsar progenitors of long duration bursts predict that they are more likely to reside in metal poor environments (Woosley 1993). Low metallicities in the surrounding medium of GRBs are in general measured by absorption line studies of GRB afterglows at $z \gtrsim 2$ (Savaglio et al. 2003; Vreeswijk et al. 2004; Chen et al. 2005; Dessauges-Zavadsky et al. 2006; Prochaska et al. 2007). This technique is used mostly to infer iron group elements along the line of sight towards the GRB. High resolution spectra of GRB afterglows have

\footnotetext{
${ }^{2}$ http://www.astro.ku.dk/ pallja/GRBsample.html
} 


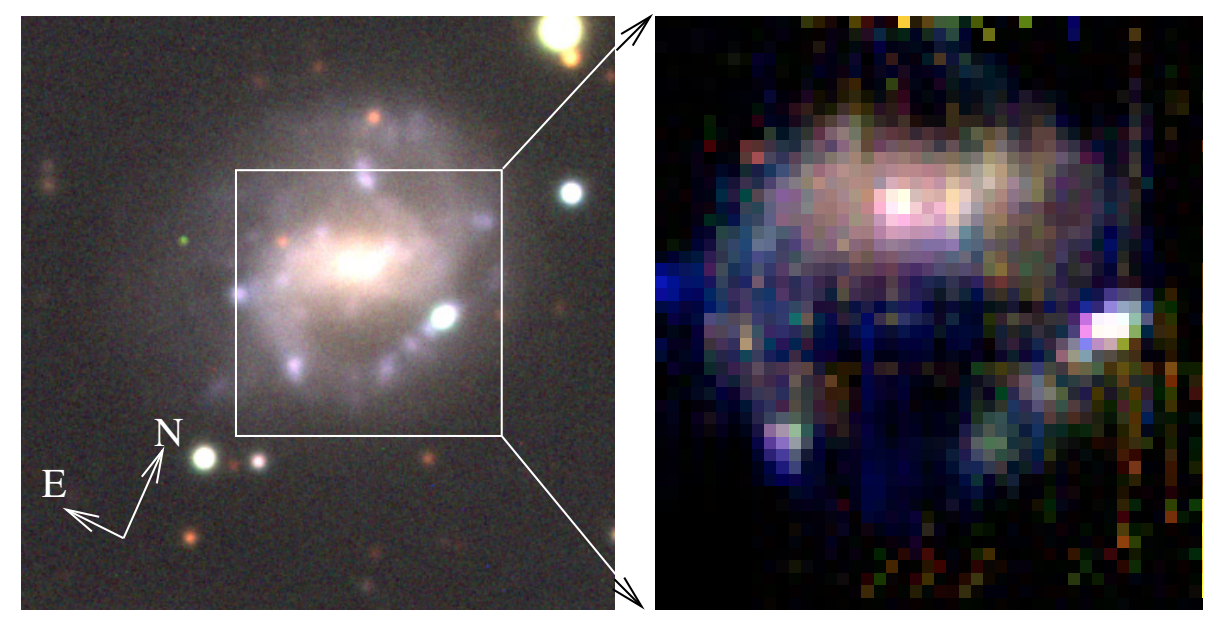

Fig. 1. Colour composite images of the host galaxy from archive FORS BVR broad band images (left) and broad band images extracted from the VIMOS data cube (right). Each of the pixels in the VIMOS image has a corresponding spectrum. The WR region is the brightest and bluest one to the West of the centre, and the SN region is seen as a fainter extension adjoining this region to the South. The size of the VIMOS image is $30^{\prime \prime}$ on a side, corresponding to $5.3 \mathrm{kpc}$ at the redshift of the galaxy. See the electronic edition of the Journal for a colour version of this figure.

indicated that the medium probed by the spectra resides at distances larger than several tens of pc from the burst site (Prochaska et al. 2006; Chen et al. 2007), and a detailed study of one burst (GRB 060418) discovered that the environment probed by the absorption lines was at a distance of $1.7 \mathrm{kpc}$ from the burst site (Vreeswijk et al. 2007). The GRB afterglow does therefore not probe the medium immediate surrounding the burst itself, but rather the interstellar medium of the host; this implies that perhaps we are unable to estimate the metallicity of the progenitor directly using this technique.

Since the hosts of long duration bursts are undoubtedly starforming galaxies, the emission lines from the integrated optical spectra can be used to infer the metallicities provided that the redshift of the GRB is less than approximately one where the strong lines are visible in the optical. This technique is generally used to derive the oxygen abundance. Diagnostics of strong emission lines for the hosts of GRB 980425, GRB 020912, GRB 030329, GRB 031203, and GRB 060218 have indicated sub solar metallicities (Prochaska et al. 2004; Hammer et al. 2006; Gorosabel et al. 2005; Sollerman et al. 2005; Wiersema et al. 2007), and systematically lower host galaxy metallicities than found in nearby ordinary type Ic supernovae sites (Modjaz et al. 2008). It is, however, unclear how measurements derived from the observed integrated GRB host spectra relate to the overall host properties, or to the site in which the GRB exploded. If the host galaxy is clearly extended, an analysis of the spatially resolved emission lines can uncover the characteristics of the precise GRB region, or alternatively the specific environment conditions required to make a GRB. Thöne et al. (2008) investigated the spectra of the host of GRB 060505, and found that the GRB site was the youngest and most metal poor of all regions probed by the slit spectrum.

For the host of GRB 980425/SN 1998bw (ESO 184-G82), a dwarf SBc galaxy, the SN occurred in a star-forming region in the spiral arm. The region was identified by high spatial resolution images acquired with HST/STIS (Fynbo et al. 2000) and confirmed by the observation of a fading source in this region (Sollerman et al. 2002). The location of the SN was offset by a projected distance of $0.8 \mathrm{kpc}$ from the strongest starforming region in the host, which had distinct spectral signatures of Wolf-Rayet stars (Hammer et al. 2006). The spectrum of the $\mathrm{SN}$ region did not have any detected WR features. Other
GRB hosts at $z \lesssim 0.1$ did not show clear signatures of WR stars in emission either (Margutti et al. 2007; Wiersema et al. 2007). At higher redshifts, the absence of C IV $\lambda 1550$ absorption lines in winds of WR stars can be explained by the afterglow, which ionises the surrounding medium to a large radius (Chen et al. 2007, but see also Starling et al. 2005).

All previous analyses of emission lines from GRB hosts were limited to regions within the slit. Hence, the data could be affected by slit-losses and leading to an under-estimated integrated star formation rate (SFR). In this paper, we present data for the GRB 980425 host galaxy obtained with integral field spectroscopy. The data from the VIMOS integral field unit (IFU) presented in Sect. 2 allows us to study a significant fraction of the surface of the host with a resolution of $0.27 \mathrm{kpc}$, and to analyse most of its high surface brightness regions (see Fig. 1). We use the data to compile maps of emission lines and their ratios and determine the reddening, SFRs, specific SFRs, metallicities, densities, temperatures, and kinematic and stellar masses, as presented in Sect. 3. We compare the emission line ratios with those of other GRB hosts in Sect. 4, and show evidence that, while abundances may vary, the regions are in all cases very young. In Sect. 5, we discuss the implications for the unresolved high redshift GRB hosts. We adopt a cosmology with $H_{0}=70 \mathrm{~km} \mathrm{~s}^{-1} \mathrm{Mpc}^{-1}$ throughout the paper.

\section{Observations and data reduction}

The host galaxy was observed on six different clear or photometric nights in April and May 2006 in service mode at the Very Large Telescope UT3 Melipal with the VIMOS integral field spectroscopic mode. The high spectral resolution setup of VIMOS consists of four quadrants of 400 spectra each, recorded on 4 CCDs. The field of view (FOV) when using the 0.67 lens array is $27^{\prime \prime} \times 27^{\prime \prime}$. The size of the host as measured in FORS images obtained from the ESO archive (64.H-0375 and 66.D-0576(A), PI: F. Patat), is about $1^{\prime}$ in diameter, while the high surface brightness emission is roughly half that size. We obtained a total integration time of five hours in three different settings as listed in Table 1. Since the host galaxy emission occupies the entire IFU FOV (see Fig. 1), separate 200 s observations of the sky offset by 2 arcmin were used for sky background subtraction. The wavelength coverage of the VIMOS data from the three settings allowed us to cover all of the strong emission lines 
Table 1. Log of the observations. $R$ gives the average spectral resolution as measured from the data. The resolution varies by $10 \%$ from one spectrum to the next. The seeing is the one recorded by the observatory DIMM monitor, and not the effective spatial resolution obtained from the data cube.

\begin{tabular}{lclccc}
\hline \hline Date & $\begin{array}{c}\text { Integration } \\
\text { time }(\mathrm{s})\end{array}$ & Setting & $\begin{array}{c}\lambda \\
(\AA)\end{array}$ & $\begin{array}{c}R \\
(\lambda / \Delta \lambda)\end{array}$ & $\begin{array}{c}\text { Seeing } \\
\left({ }^{\prime \prime}\right)\end{array}$ \\
\hline 2006 April 25 & $3 \times 600$ & LR blue & $3600-6800$ & 250 & 0.9 \\
2006 May 24 & $2 \times 600$ & LR blue & $3600-6800$ & 250 & 0.9 \\
2006 April 29 & $3 \times 1800$ & HR orange & $5045-7475$ & 2700 & 0.7 \\
2006 May 3 & $2 \times 1800$ & HR orange & $5045-7475$ & 2700 & 0.8 \\
2006 May 24 & $2 \times 1800$ & HR blue & $3970-6230$ & 2600 & 1.0 \\
2006 May 25 & $1 \times 1800$ & HR blue & $3970-6230$ & 2600 & 0.4 \\
\hline
\end{tabular}

used for abundance determinations. The low spectral resolution data also covered the [O II] $\lambda 3727$ line. At the redshift of the host, this emission line is detected at the observed wavelength of $3759 \AA$, where the transmission of the VIMOS IFU is about 10 times lower than its maximum value.

The data were reduced with the reduction package P3d (Becker 2002) and the following steps. After bias subtraction, the location of the 1600 spectra and the traces were identified using continuum lamp spectra obtained immediately after the science observations. Arc line spectra taken after science exposures were extracted. The science spectra were extracted, wavelength calibrated, and flat fielded using the (wavelength dependent) transmission function determined from the continuum spectra. The calibrated science data were arranged in a 3D data cube. Each data cube was corrected for atmospheric extinction. The sky background was subtracted using separately reduced sky background data cubes.

The 4 quadrants of the combined data cubes were flux calibrated separately using observations of spectrophotometric stars placed in each of the four quadrants. Spatial shifts between the individual data cubes were determined and the cubes were combined, using routines similar to standard image combination procedures in IRAF, while masking out the dead spectra that were located mainly at the edge of the FOV. We ignored the differential atmospheric refraction effects, since the observations were mostly taken at air masses lower than 1.4. This should theoretically produce a maximum shift of $\sim 1$ spaxel (a spaxel "spatial picture element", is defined to be a single spectrum in the array) between the blue and the red end of the spectral range (Filippenko 1982). However, the maximum spatial shift in the data cubes was measured to be less than 0.2 pixels by a cross correlation technique. Since we used dithered pointings that were offset by $3^{\prime \prime}$, the resulting FOV in the reduced and combined data cube was $30^{\prime \prime} \times 30^{\prime \prime}$.

To check the absolute flux calibration, we re-reduced broad band FORS $B, V$, and $R$ images from the ESO archive. We then extracted the spectra for different regions from the VIMOS high spectral resolution cubes, and convolved each spectrum with the Bessell filter transmission functions to calculate the broad band magnitudes (Bessell 1990). For individual regions, such as the SN and WR region and the entire VIMOS FOV in Fig. 1, the magnitudes and colours agree with those for the FORS images to within $10 \%$ accuracy. It is impossible to apply a simple scaling to the VIMOS data cube to improve the absolute spectrophotometric calibration for all regions within the FOV. The sensitivity of the VIMOS-IFU is known to show variations depending on the exact location of the spectrophotometric standard star (see Monreal-Ibero et al. 2006), which affects the absolute flux calibration. Finally, the data cube was corrected for the foreground Galactic reddening of $E_{B-V}=0.059$ mag (Schlegel et al. 1998).
Even though the VIMOS data were taken in good seeing conditions, using the 0.'67 spatial sampling provided an effective resolution element of $1 . .5$, or $0.27 \mathrm{kpc}$ at the redshift of the galaxy.

\section{Results}

\subsection{Emission line maps and spectra}

IFU data cubes allow us to estimate fluxes both from images and spectra. Two-dimensional images are created by coadding monochromatic slices (equivalent to channel maps in the radio astronomy community) in the data cube along the wavelength direction. These can be analysed with standard imaging methods. One-dimensional spectra are created by selecting some pixels or "spaxels" in the data cube and co-adding each wavelength separately. To extract one dimensional spectra and visualize the data cubes, we used the program QFitsView created by Thomas Ott.

To estimate line fluxes, we used two methods: fitting of lines in spectra with Gaussian profiles and a simple integration of narrow-band images created from the data cube. Since the emission lines from the different regions are not strictly Gaussian in shape, we cannot rely on a fully automated line-fitting routine to extract line fluxes. To create emission line maps and derive fluxes for different regions, we use the second method, integrating over 8 slices from the data cube in the spectral direction centred on the measured wavelength $(1+z) \lambda_{\text {lab }}$. This corresponds to twice the size of the spectral FWHM and is equivalent to integrating over a wavelength interval between 4.4 and $4.7 \AA$ about the lines. The emission lines are barely resolved even with the high spectral resolution $\left(\sim 110 \mathrm{~km} \mathrm{~s}^{-1}\right.$ at $\left.5000 \AA\right)$, and no emission lines in any of the regions have widths significantly larger than the spectral resolution. The emission line fluxes determined from the two methods are consistent to within $10 \%$ accuracy.

The continuum flux was determined by fitting a first order polynomial over a region about the emission line in each of the individual spectra. The continuum region was selected free of other emission lines and examined interactively. The backgrounds were subtracted to provide the pure emission line maps. Two examples are presented in Fig. 2, which shows the H $\alpha$ emission line map (left) and [O III] $\lambda 4363$ (right). Only the brightest $\mathrm{H}$ II region in the host shows detectable emission from the latter, faint, temperature-sensitive line. This region also shows emission lines with characteristic Wolf-Rayet features (Hammer et al. 2006). We investigated the emission line maps to look for WR emission lines (He or $\mathrm{N}$ lines) in other regions, but these lines are below the detection limit in the data cube. Following Hammer et al. (2006), the brightest region is denoted "WR", while the star-forming region in which the SN/GRB occurred is denoted "SN". The SN occurred in an H II region that is 

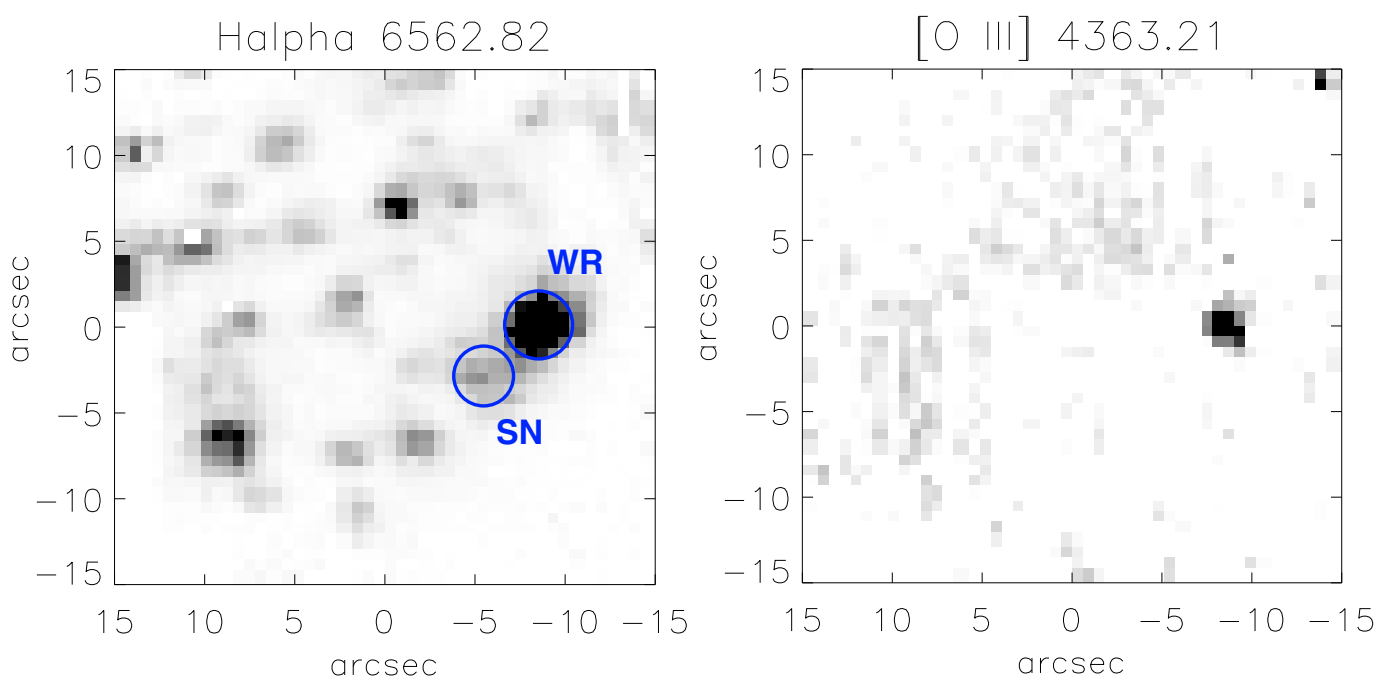

Fig. 2. Maps of a typical strong emission line (left: $\mathrm{H} \alpha$ ), and a faint one (right: [O III] $\lambda 4363$ ). The region that is bright in [O III] is the only region where emission lines from WR stars are detected. The circles have a radius of 2 ". 5 corresponding to $440 \mathrm{pc}$.

located 5 arcsec to the South-East of the centre of the WR region, or $800 \mathrm{pc}$ in projection at the redshift of the host. In the spectrum extracted from the WR region, we identify 45 different lines within the spectral coverage ${ }^{3}$. In other regions, the number of detectable lines is significantly smaller. This paper considers mostly strong emission lines, since these can be identified throughout the host galaxy, allowing a fair comparison between different regions.

\subsection{Emission line fluxes}

Table 2 lists the line fluxes and derived properties of several distinct and spatially resolved $\mathrm{H}$ II regions identified in the $\mathrm{H} \alpha$ map. Most regions listed in the table are labeled with their coordinates relative to the $\mathrm{H} \alpha$ map. A significant fraction (34\%) of the integrated emission line flux within the VIMOS FOV originates in the WR region. The final row in the Table presents the sample mean and standard deviation as a measure of the spread of values in the different $\mathrm{H}$ II regions.

The $\mathrm{H} \alpha$ and $\mathrm{H} \beta$ Balmer emission line fluxes have been corrected for an underlying stellar absorption component with the equivalent width $E W(\mathrm{H} \alpha) \approx E W(\mathrm{H} \beta)=2.6 \AA$, which is an appropriate value for young stellar populations (González Delgado et al. 1999). We observe no evidence for an absorption line about the $\mathrm{H} \beta$ line in any individual spaxel. While the absorption line should be present in any stellar population, the non-detection in individual spectra is because the continuum emission is faint and the signal-to-noise ratio in each spaxel is typically only a few. In the combined galaxy spectrum an underlying stellar absorption component is evident with an equivalent width of $\sim 2 \AA$.

The equivalent width is defined as $E W=\int_{\lambda}\left(f_{\text {line }, \lambda} / f_{\text {cont }, \lambda}-\right.$ 1)d $\lambda$, where $f_{\text {line }, \lambda}$ is the measured emission line flux and $f_{\text {cont }, \lambda}$ is the continuum flux. We correct the integrated line flux for an absorption of $2.6 \AA$ such that $f_{\text {line,cor }}=f_{\text {line }}+2.6 \times f_{\text {cont }}$. For regions with fainter Balmer emission line fluxes and lower equivalent widths, the correction to the $\mathrm{H} \beta$ line can be as substantial as $15 \%$, relative to a typical correction of a few percent for the brightest lines. This implies that the extinction will be over estimated and the metallicity under estimated, if this correction is not applied. For the brightest emission line regions the corrections are small.

The [O II] emission line in the low spectral resolution data has considerably larger uncertainty than the other bright emission lines. Furthermore, no spectrophotometric standard star was observed with the low spectral resolution setup at the dates of the observations. Instead, we used standard star observations acquired at times that differed by about one week, and relied on the fact that the overall shape of the transmission function of VIMOS is stable with time. To derive the emission line fluxes, we compared the extracted $\mathrm{H} \beta$ fluxes for the $\mathrm{H}$ II regions and applied a scaling to the [O II] emission line.

If we compare the line fluxes of the WR and $\mathrm{SN}$ regions with those reported for long slit spectra in the literature (Sollerman et al. 2005; Hammer et al. 2006), as well as FORS spectra obtained from the ESO archive (Program ID 275.D-5039(A), PI: Patat), we must take into account the different spatial regions sampled. Due to different effects, such as seeing-dependent slit losses, an uncertain placement of the slit, different spectral resolutions, seeing, as well as reduction procedures to extract the one dimensional spectra, it is difficult to compare directly our extracted fluxes with values in the literature (e.g. Sollerman et al. 2005; Hammer et al. 2006). As an example, the fluxes from the literature agree with our results obtained from the archive FORS long slit spectrum only to within a factor of approximately 2 . For these reasons, we cannot compare the VIMOS spectra with those derived from long slit spectra. While slit losses affect the long slit spectra, the ratios of the emission line fluxes should remain the same as in the IFU data provided that the same region is extracted. The $\mathrm{H} \alpha / \mathrm{H} \beta$ emission line ratio is used frequently to estimate the extinction (see Sect. 3.5) and in the $\mathrm{SN}$ region this ratio is used to infer both zero extinction (Sollerman et al. 2005) and $A_{\mathrm{V}}=1.02$ (Hammer et al. 2006), while we find $A_{\mathrm{V}}=1.95$ in the IFU data, similar to $A_{\mathrm{v}}=1.73$ in Savaglio et al. (2008). For other emission line ratios of lines with similar wavelengths, we find differences of up to a factor of two. This could be due to spectrally blended lines or blending of separate $\mathrm{H}$ II regions due to seeing.

\footnotetext{
3 The spectrum presented in Hammer et al. (2006) also has significantly more lines than listed in the paper.
} 


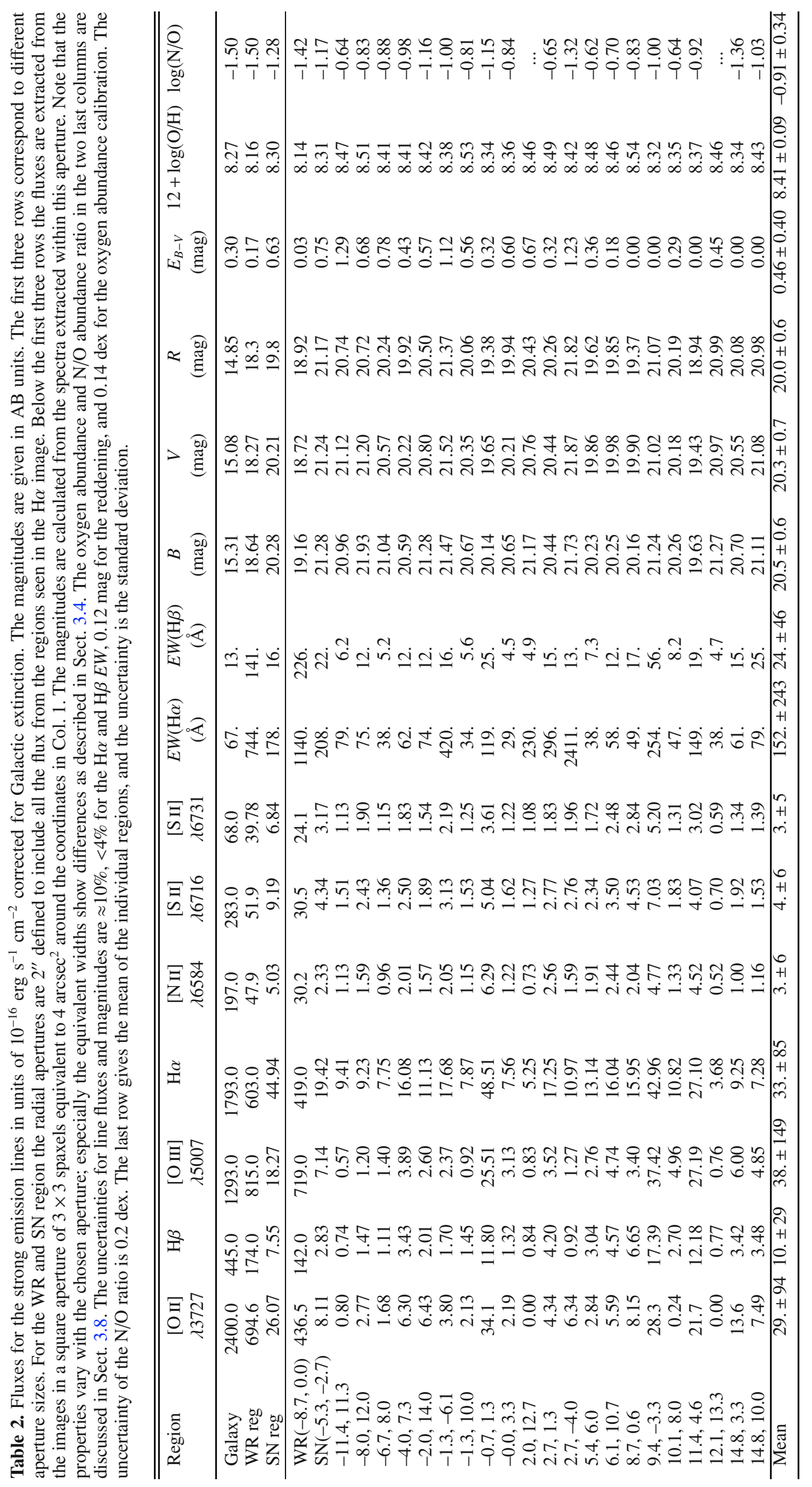


The broad band magnitudes derived for these regions agree with those for the FORS imaging data to within 5-10\%, which we take as representative of the uncertainty in the emission line fluxes. This uncertainty is significantly higher than the pure statistical uncertainty derived from the number counts extracted for each line and region in the non-flux calibrated data $(<3 \%$ for the faintest lines in Table 2). The uncertainties in the $\mathrm{H} \alpha$ and $\mathrm{H} \beta$ equivalents width are $14 \%$ since they are derived from a ratio of two quantities each with an uncertainty of $10 \%$. The uncertainties of the reddening and abundances are derived by propagating errors. The uncertainties of all reddening values are $0.12 \mathrm{mag}$. Propagating the flux errors for the abundances determination (see Sect. 3.8), the uncertainty is only 0.03 for the oxygen abundances. Since the scatter in the abundance diagnostics used to derive the oxygen abundance itself is 0.14 dex (Pettini \& Pagel 2004), this will dominate the overall uncertainty.

Similarly, taking into account a wide range of relevant temperatures to derive the $N$ abundance we find a 0.2 dex uncertainty in the $\mathrm{N} / \mathrm{O}$ ratio.

For the oxygen abundance derived in Sect. 3.8, we use the solar value $12+\log (\mathrm{O} / \mathrm{H})=8.66$ (Asplund et al. 2004).

\subsection{Kinematics}

Although the lines are not exactly Gaussian in shape, we use an automated line-fitting routine to estimate the peak position of the $\mathrm{H} \alpha$ line and derive the velocity structure of the galaxy presented in Fig. 3. The zero velocity is defined for the geometric centre of the galaxy. The uncertainty in the wavelength calibration for each spaxel is less than $0.2 \AA$, which corresponds to a velocity uncertainty of $10 \mathrm{~km} \mathrm{~s}^{-1}$.

To derive the kinematic mass of the entire galaxy, it is necessary to study the rotation curve out to galactocentric radii at which it flattens, which correspond to the outskirts of the disk. In the velocity map, there is a gradient that increases all the way to the edge, so the estimated kinematic mass is limited to within $15^{\prime \prime}$ or correspondingly $2.6 \mathrm{kpc}$ in radius. We model the rotation of the galaxy by assuming simple rotation of a solid disk. This is appropriate at least for the central part of the galaxy because of the presence of a bar in the centre. The only free parameters are the centre of the disk, the position angle, and the velocity. The model velocity field is subtracted from the observations, and the model with the smallest velocity residuals are taken to present the best fit. A best-fit model of the velocity field within the VIMOS FOV provides a maximum rotation velocity of $25 \mathrm{~km} \mathrm{~s}^{-1}$ at this distance. With these values, the mass is $4 \times 10^{8} /(\sin i)^{2} M_{\odot}$, where $i$ is the inclination of the galaxy. For $i \approx 50^{\circ}$ estimated from surface photometry of the high surface brightness emission in the FORS $B$ band image, the lower limit to the galaxy mass is $6 \times 10^{8} M_{\odot}$ within the VIMOS FOV. The largest uncertainty originates from the estimate of the inclination: most of the galaxy does not have a high surface brightness, and this could produce an underestimation of the mass by a factor of $\sim 5$. The total galaxy mass derived from a $K$ band image $\left(1.1 \times 10^{9} M_{\odot}\right)$ corresponds to a stellar mass of about twice the value within the VIMOS FOV (Castro Cerón et al. 2008). If the inclination axis is lower than $50^{\circ}$, the ratio of the total mass indicated from the velocity to the stellar mass increases correspondingly, i.e. the dark matter halo mass increases.

The estimated mass is consistent with the mass-luminositymetallicity relation for SDSS galaxies (Tremonti et al. 2004), which predicts that $12+\log (\mathrm{O} / \mathrm{H})=8.5 \pm 0.2$ for a galaxy of this (stellar) mass. The dynamical mass estimate, derived

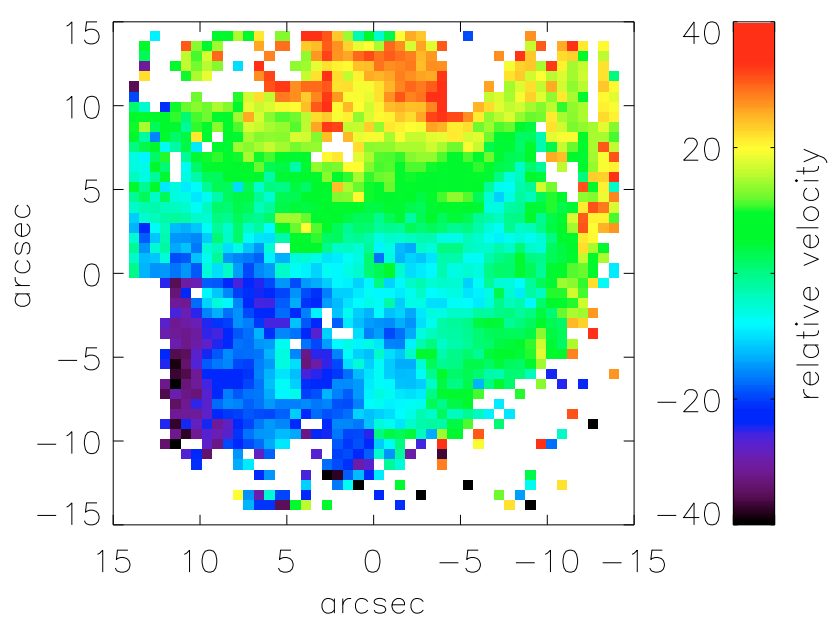

Fig. 3. Velocity map derived from fits to the $\mathrm{H} \alpha$ emission line in all spaxels. The values are in units of $\mathrm{km} \mathrm{s}^{-1}$. See the electronic edition of the Journal for a colour version.

above, includes both stellar and dark matter: a measurement of the stellar mass only should correspond to a lower expected abundance. In Sect 3.8, we measure an oxygen abundance of $12+\log (\mathrm{O} / \mathrm{H})=8.3$, which corresponds to a relatively small mass galaxy (see also Table 2).

\subsection{Equivalent widths}

The continuum around the $\mathrm{H} \alpha$ and $\mathrm{H} \beta$ lines was calculated to determine the equivalent widths $(E W \mathrm{~s})$ corrected for the underlying stellar absorption. The resulting maps are shown in Fig. 4. The WR region is distinctive in this image. In the WR region, the $\mathrm{H} \alpha$ and $\mathrm{H} \beta E W \mathrm{~s}$ are $744 \AA$ and $141 \AA$, respectively, within a 2 arcsec radial aperture, while in the SN region the $E W \mathrm{~s}$ are $178 \AA$ and $16 \AA$, respectively. The $\mathrm{H} \alpha$ value for the $\mathrm{SN}$ region is higher by a factor of two than the measurement of Hammer et al. (2006); this disagreement is probably caused by aperture effects in long slit spectra or differences in the size of the extracted regions. The fewer the number of extracted spaxels the larger the $E W \mathrm{~s}$. As an example, the $E W$ in the brightest spaxel in the WR region has an $\mathrm{H} \alpha E W$ of $1600 \AA$. According to instantaneous burst models from Starburst99 (Leitherer et al. 1999), this corresponds to an age of 3.1 Myr. Similarly, the brightest spaxel of the $\mathrm{SN}$ region has an $\mathrm{H} \alpha E W$ of $320 \AA$, corresponding to an age of 5.0 Myr. If we analyse the $E W \mathrm{~s}$ for the other $\mathrm{H}$ II regions in the host, we find that the $\mathrm{SN}$ region is more representative of the entire host galaxy, while the WR region is the youngest.

\subsection{Intrinsic reddening}

The ratio of the maps $\mathrm{H} \alpha / \mathrm{H} \beta$ provides a measure of the reddening by dust. In the Case $\mathrm{B}$ recombination scenario, the theoretical dust free ratio is 2.85 for a temperature of $10000 \mathrm{~K}$ (Osterbrock 1989). The reddening is calculated by the equation $E_{B-V}=1.98\left[\log _{10}\left(\mathrm{H} \alpha_{\text {obs }} / \mathrm{H} \beta_{\text {obs }}\right)-\log _{10}(2.85)\right]$. The factor 1.98 assumes a Milky Way type extinction law (Fitzpatrick 1999), but choosing a different extinction curve does not change this factor significantly. The reddening map is presented in Fig. 5. We adopted a threshold for the $\mathrm{H} \alpha$ flux of $3 \times 10^{-17} \mathrm{erg} \mathrm{s}^{-1} \mathrm{~cm}^{-2}$ to calculate the extinction, because in regions where the flux is lower, the value of $E_{B-V}$ becomes correspondingly uncertain. 

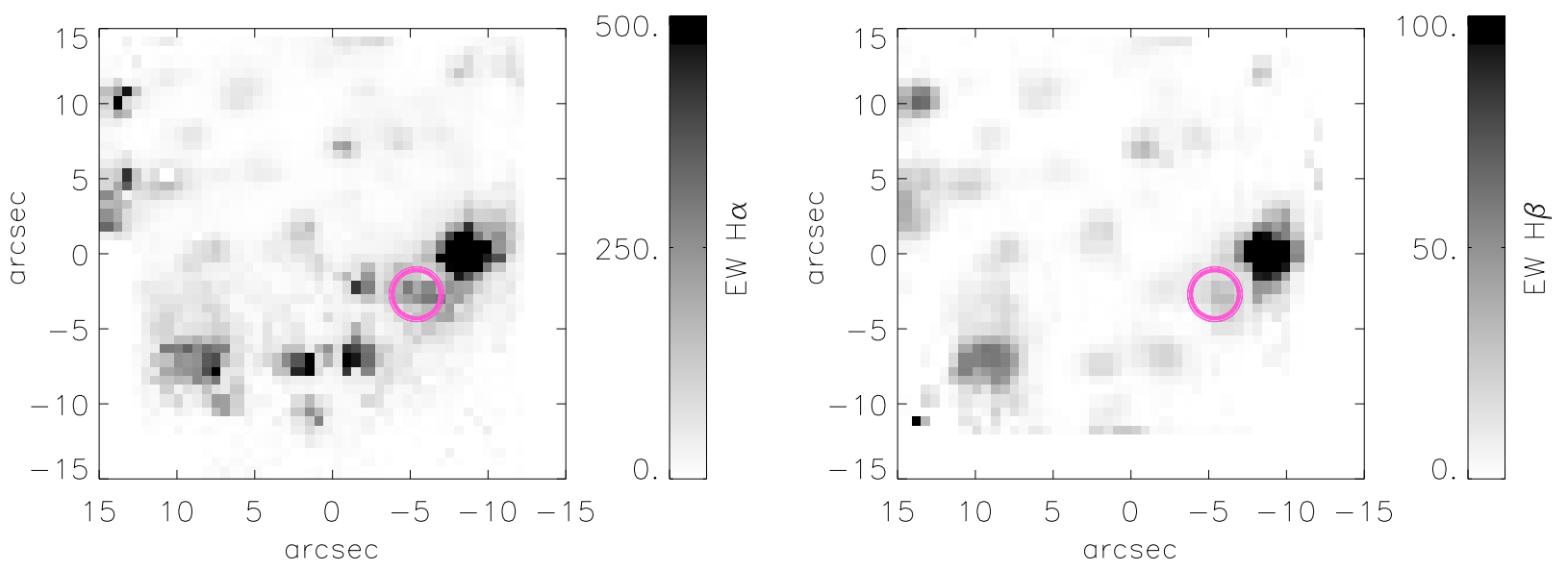

Fig. 4. Map of the $\mathrm{H} \alpha E W$ (left panel) and map of the $\mathrm{H} \beta E W$ (right panel). The noise pattern in the lower middle part of the $\mathrm{H} \alpha E W$ map is caused by noise in the continuum flux estimate in the VIMOS data. In this region, the surface brightness is low (see Fig. 1). Both panels show that the WR region has the largest $E W$ over the face of the galaxy and indicates that this is the youngest star-forming region. The circle marks the position of the SN.

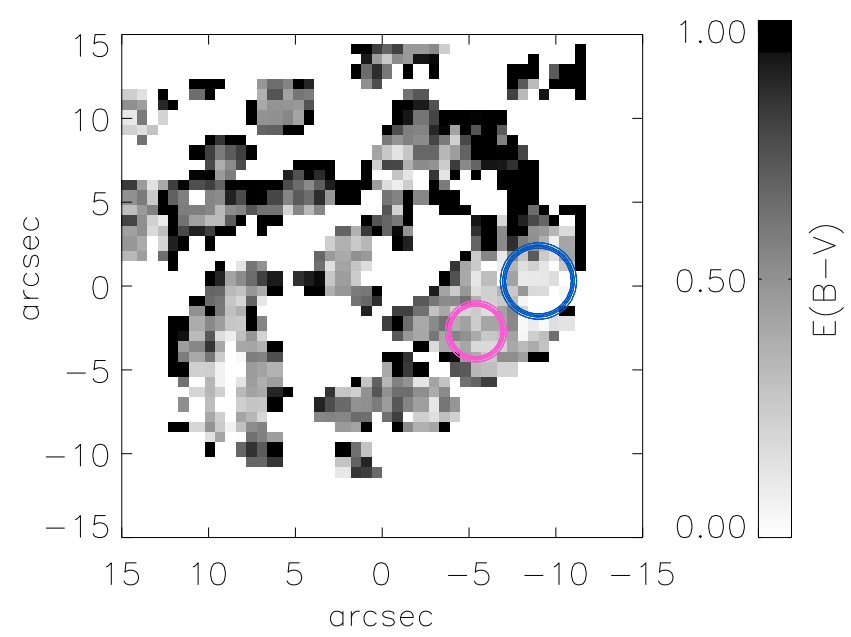

Fig. 5. Reddening map, showing the value of $E_{B-V}$. Half of the WR region at coordinates $(-8.7,0)$ appears relatively free of dust (white colour), while the $\mathrm{SN}$ region has $E_{B-V}=0.63 \mathrm{mag}$. The small circle indicates the location of the $\mathrm{SN}$ region, and the larger circle the location of the WR region. Apart from the white colour in the WR region, white regions in this and following maps represent spaxels where the $\mathrm{H} \alpha$ flux is smaller than $3 \times 10^{-17} \mathrm{erg} \mathrm{s}^{-1} \mathrm{~cm}^{-2}$.

Whereas the host has an overall $E_{B-V}=0.30 \mathrm{mag}$, approximately half of the WR region appears to be dust free with an overall average of $0.17 \mathrm{mag}$. On the other hand, the $\mathrm{SN}$ region appears to have significantly more reddening with an estimated average of $E_{B-V}=0.63$ mag. A similar analysis can be completed using the $\mathrm{H} \beta / \mathrm{H} \delta$ emission line ratio but this ratio has a larger uncertainty due to the fainter emission lines involved. The results obtained from this ratio agree with those for $\mathrm{H} \alpha / \mathrm{H} \beta$ to within the uncertainties. Using the tight correlation between the $\mathrm{H} \alpha$ and $24 \mu \mathrm{m}$ luminosities (Kennicutt et al. 2007) and the IR luminosity of $2.34 \times 10^{40} \mathrm{erg} \mathrm{s}^{-1}$ for the WR region (Le Floc'h et al. 2006), we measure an overall $3 \%$ extinction correction of the $\mathrm{H} \alpha$ flux. The spatial resolution of Spitzer is insufficient to resolve separately the $\mathrm{SN}$ region.

The reddening inferred from the FORS archive spectrum is $E_{B-V}=0.16$ and $0.14 \mathrm{mag}$ for the WR and SN region, indicating that a significant discrepancy exists only for the $\mathrm{SN}$ region.
For this region, Sollerman et al. (2005) measured $E_{B-V}=0$ mag, while Hammer et al. (2006) found $E_{B-V}=0.39$ mag and Savaglio et al. (2008) found $A_{\mathrm{V}}=1.73$. If the extinction in the SN region is as high as we find, this would imply that the supernova was exceptionally (and unreasonably) bright and blue. If the extinction is larger than inferred from observations of SN 1998bw, this could imply that the SN was in the foreground of a dustier H II region.

The magnitudes of the $\mathrm{SN}$ region in the FORS images and the VIMOS data agree to within 0.05 mag when the same aperture size is used. Because the long slit data cannot be used to calculate the magnitude as a consistency check, we assume that the large extinction found in the VIMOS data is correct, even though there is a significant disagreement with some literature values.

\subsection{Star formation rates}

The $\mathrm{H} \alpha$ emission line flux can be converted to a SFR given the transformation in Kennicutt (1998). This law was derived for complete galaxy disks, and may be inappropriate for individually resolved $\mathrm{H}$ II regions and very young regions experiencing almost instantaneous bursts of star formation. The ages derived for individual H II regions in the GRB 1998bw host are similar, and the $\mathrm{H} \alpha$ luminosities are sufficiently high; we can therefore assume that the conversion of $\mathrm{H} \alpha$ luminosity to SFR is applicable to individual regions (see also Kennicutt et al. 2007). A map of the SFR surface density is shown in Fig. 6.

As derived from the $\mathrm{H} \alpha$ emission line luminosity, the total SFR within the FOV is $0.21 M_{\odot} \mathrm{yr}^{-1}$. From the integrated onedimensional spectrum over the entire FOV, we measure $S F R=$ $0.23 M_{\odot} \mathrm{yr}^{-1}$. This difference is caused by differences in the uncertainties in estimating the underlying continuum: within the image, the continuum is estimated in each spaxel, while the continuum is measured in only one dimension in the extracted spectrum. Within the 30 arcsec square aperture, the $\mathrm{H} \alpha$ narrow-band image obtained by Sollerman et al. (2005) provides a measurement of $0.22 M_{\odot} \mathrm{yr}^{-1}$ (corrected for $10 \%$ flux from [N II] emission present in the narrow band too).

At $z \sim 1$, GRB host galaxies have typical UV-based specific star formations rates (SSFRs) of about $10 M_{\odot} \mathrm{yr}^{-1}\left(L / L^{*}\right)^{-1}$, 

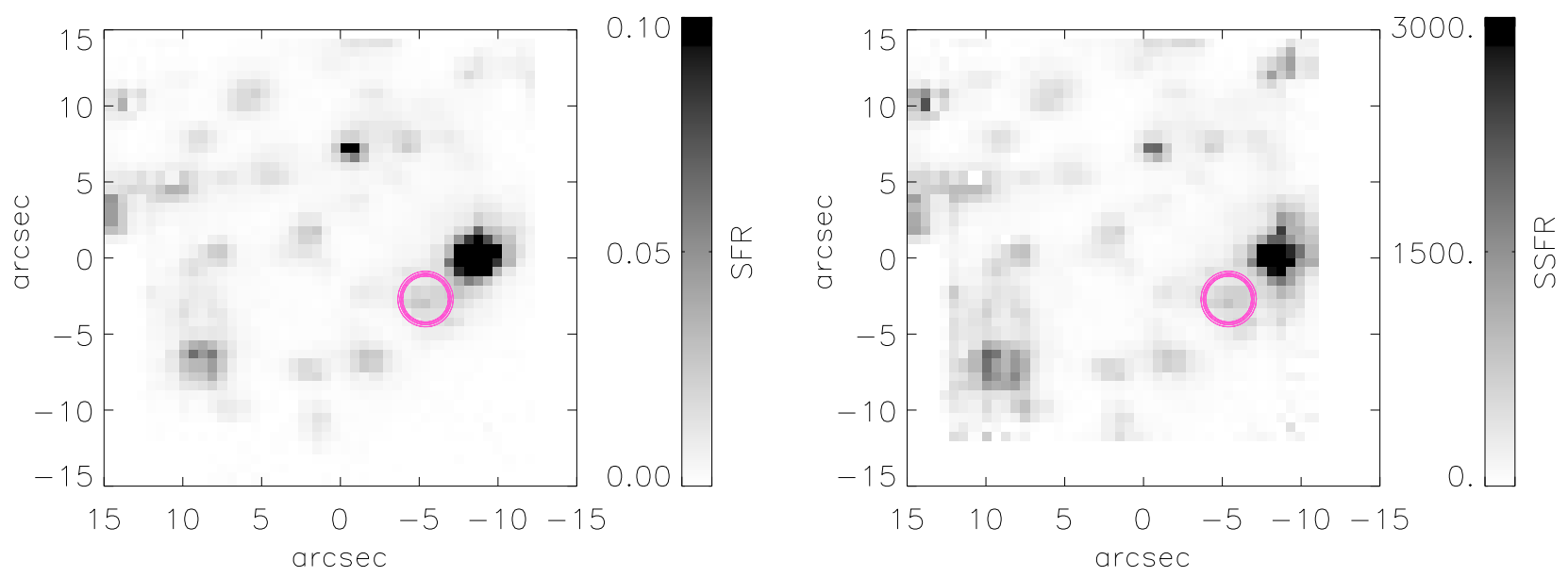

Fig. 6. Left panel: map of the SFR converted from the $\mathrm{H} \alpha$ map. The units are in $M_{\odot} \mathrm{yr}^{-1} \mathrm{kpc}^{-2}$. Right panel: map of the luminosity specific SFR in units of $M_{\odot} \mathrm{yr}^{-1}\left(L / L^{*}\right)^{-1} \mathrm{kpc}^{-2}$ (uncorrected for intrinsic extinction in the host). The WR region has the largest value over the face of the host. The circle marks the location of the SN region.

where the luminosities $L$ are normalised to the $B$ band luminosity $L^{*}$ corresponding to the magnitude $M^{*}=-21$ (Christensen et al. 2004a). To determine the SSFR of the GRB 980425 host, the spectra in the blue high resolution cube are convolved with a Bessell transmission function (Bessell 1990) to derive the $B$ band magnitude in each spaxel. The SFR map is then divided by the relative luminosity $L / L^{*}$ as shown in the right panel of Fig. 6, which measures the $\mathrm{H} \alpha$ flux relative to the blue continuum luminosity. In the WR region, the $\mathrm{H} \alpha$ flux is much larger for a given continuum flux than in other regions.

Since the SFR measurement is more relevant on a galaxy wide scale, we also analyse the integrated properties of the galaxy. The integrated magnitude in the VIMOS data is $m_{B}=$ $15.3 \mathrm{mag}$, corresponding to an absolute magnitude of $M_{B}=$ -17.5 , and $S S F R=5.3 M_{\odot} \mathrm{yr}^{-1}\left(L / L^{*}\right)^{-1}$. This is slightly smaller than the UV-based SSFRs of GRB hosts at higher redshift. The entire GRB host has a $S S F R \approx 7 M_{\odot} \mathrm{yr}^{-1}\left(L / L^{*}\right)^{-1}$ (Sollerman et al. 2005). If we apply a correction for the internal extinction in the host $\left(E_{B-V}=0.3\right)$ we derive $S S F R=10.2 M_{\odot} \mathrm{yr}^{-1}\left(L / L^{*}\right)^{-1}$.

The high value for the WR region is distinctive compared to that of GRB hosts at $z \sim 1$. However, the explanation is that the individual star forming regions are resolved (here normalised to $\mathrm{kpc}^{-2}$ ), while the higher redshift hosts are investigated using integrated properties. Other lower redshift GRB host with high SSFRs include the GRB 030329 host with $\sim 25 M_{\odot} \mathrm{yr}^{-1}\left(L / L^{*}\right)^{-1}$ (Gorosabel et al. 2005), and the GRB 031203 host with SSFR $39 M_{\odot} \mathrm{yr}^{-1}\left(L / L^{*}\right)^{-1}$ (Sollerman et al. 2005). All of these values are derived from emission line based SFRs, and it is known that there are discrepancies between the SFRs determined from the UV flux and from emission lines, albeit the discrepancies found are typically lower by a factor of less than 2 . Nevertheless, the high values for GRB hosts are unusual compared to field galaxies, which have typical UV continuum based $S S F R \lesssim$ $5 M_{\odot} \mathrm{yr}^{-1}\left(L / L^{*}\right)^{-1}$ (Christensen et al. 2004a).

\subsection{Electron temperature and densities}

Ideally, the temperatures and densities of the $\mathrm{H}$ II regions should be derived by including faint nebular emission lines such as the [O III] $\lambda 4363$, or the [N II] $\lambda 5755$ line (Osterbrock 1989). Only for one region do we detect the temperature sensitive [O III] $\lambda 4363$ line (right panel in Fig. 2). In the FORS data, we also detect the faint line only in the WR region. From the ratio $\mathrm{O} 3=(4959+5007) / 4363$ we find that $T=10300 \pm 1000 \mathrm{~K}$ for the WR region. In contrast, Hammer et al. (2006) measured temperatures for the SN and WR region of between 11000 and $12000 \mathrm{~K}$.

To determine the electron densities, we analyse the ratio of the [S II] $\lambda \lambda 6717,6731$ lines using the IRAF package NEBULAR, which is based on the code presented by Shaw \& Dufour (1995). At the spectral resolution of VIMOS $(R \sim 2900$ as measured from sky lines), the sulfur lines are clearly resolved, while at the lower resolution of the FORS spectrum $(R=650)$ the lines are blended. The WR region has a density of $120 \mathrm{~cm}^{-3}$ derived from the $[\mathrm{S} \mathrm{II}]$ line ratio, which for most regions is close to 1.3. Since the flux calibration does not affect the uncertainty in the ratio, the error for the ratio is 0.03 based on the number counts in the spectra. A density of the order $100 \mathrm{~cm}^{-3}$ is typically derived for other GRB hosts (Prochaska et al. 2004; Wiersema et al. 2007; Savaglio et al. 2008).

\subsection{Abundances}

We use the O3N2 abundance diagnostics from Pettini \& Pagel (2004) to determine the oxygen abundance. This diagnostics involve the line flux ratios [O III] $\lambda 5007 / \mathrm{H} \beta$ and [N II] $\lambda 6586 / \mathrm{H} \alpha$, whose lines are close in wavelength so that extinction plays a minor role. Figure 7 shows the metallicity map of the host galaxy and illustrates the values in Table 2 in the units $12+\log (\mathrm{O} / \mathrm{H})$, with the solar value of 8.66 adopted from Asplund et al. (2004). Extracting the one-dimensional spectra of the WR and SN regions yields $12+\log (\mathrm{O} / \mathrm{H})=8.16$ and 8.30 , respectively, which corresponds to 0.32 and 0.44 solar abundance. The uncertainties in the abundance is $0.03 \mathrm{dex}$ if we propagate the uncertainties in the emission line fluxes. In comparison, the $1 \sigma$ scatter uncertainty of 0.14 dex for data points in Pettini \& Pagel (2004) used to derive the $\mathrm{O} 3 \mathrm{~N} 2$ diagnostics is clearly far higher. The calibration is derived for values of $\mathrm{O} 3 \mathrm{~N} 2<1.9$, which is valid for all regions in the GRB host. Considering the large scatter of the calibration, the derived metallicities in Table 2 are similar to within a margin of error of $3 \sigma$ and the $\mathrm{WR}$ and $\mathrm{SN}$ region metallicities are consistent to within a margin of error of $1 \sigma$.

While the value for the WR region is lower than for other regions, it is also striking that the $\mathrm{SN}$ region has the second lowest metallicity; within the margins of uncertainty, many 


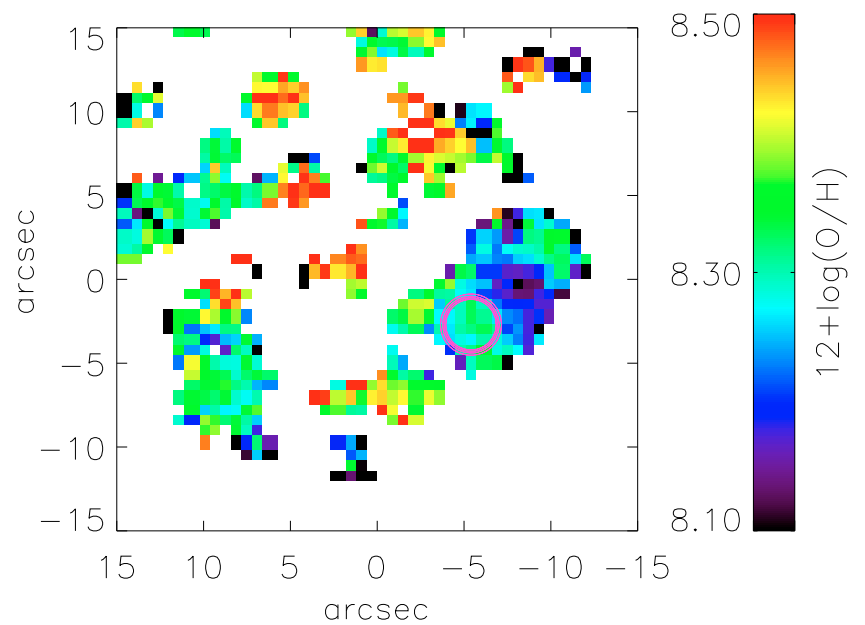

Fig. 7. Oxygen abundance map derived from the $\mathrm{O} 3 \mathrm{~N} 2$ abundance diagnostics. The WR region has the lowest abundance in the whole galaxy, and the SN region has the second lowest abundance although similar to other regions within the $1 \sigma$ uncertainty of 0.14 dex. The circle indicates the location of the SN region. (See the electronic edition of the Journal for a colour version of this figure.)

other regions, however, have the same abundance. Since these metal poor regions have brighter emission lines than the remaining regions, they dominate the integrated spectrum of the host, even though some values in Table 2 correspond to higher abundances (0.6-0.8 solar). The total galaxy spectrum has $12+\log (\mathrm{O} / \mathrm{H})=8.27(0.40$ solar $)$, i.e. similar to that of the $\mathrm{SN}$ region.

Using the N2 abundance diagnostics from Pettini \& Pagel (2004) the same values of abundances are derived to within the uncertainties, while the S2 diagnostics, which use the $\mathrm{H} \alpha /[\mathrm{S} \mathrm{II}] \lambda \lambda 6717,6731$ ratio and the $\mathrm{O} 3 \mathrm{~N} 2$ calibration in Yin et al. (2007) provides systematically higher abundances by $0.1-$ 0.15 dex for all regions. The calibration in Yin et al. (2007) was derived for a larger number of galaxies than in Pettini \& Pagel (2004), where the calibration was derived from H II regions, for which the abundances are measured using temperature sensitive lines. It is well known that different diagnostics can measure different values of abundances (Kewley \& Ellison 2008), and here we do not attempt to correct for this effect. The important point for the GRB host is not the absolute values in metallicity, but the variations of the metallicity over the galaxy image, and that one particularly bright region can dominate the measurement of the integrated abundance.

In the WR region, where the temperature sensitive [O III] $\lambda 4363$ line is detected with a flux of $\sim 5 \times$ $10^{-16} \mathrm{erg} \mathrm{s}^{-1} \mathrm{~cm}^{-2}$, the equations in Izotov et al. (2006) and the de-reddened fluxes imply an oxygen abundance of $12+$ $\log (\mathrm{O} / \mathrm{H})=8.53 \pm 0.10$, which is significantly larger than that derived for the $\mathrm{O} 3 \mathrm{~N} 2$ diagnostics. This discrepancy suggests that the $\mathrm{O} 3 \mathrm{~N} 2$ diagnostics may not be a valid approximation for this particular galaxy, and that the abundance may even be on average close to solar in contradiction to the general idea that GRB progenitors and their hosts have sub solar metallicities.

The strong emission lines in Table 2 can be used to derive the $(\mathrm{N} / \mathrm{O})$ abundance ratio. To calculate this ratio, we added the contributions to the abundance $\mathrm{O} / \mathrm{H}^{+}=\mathrm{O}^{+} / \mathrm{H}^{+}+\mathrm{O}^{2+} / \mathrm{H}^{+}$, and $\mathrm{N}$ was obtained by multiplying the $\mathrm{N}^{+} / \mathrm{H}^{+}$ratio with an ionisation correction factor using the equations in Izotov et al. (2006). The results are presented in the final column of Table 2. For the different $\mathrm{H}$ II regions, a constant temperature of $10^{4} \mathrm{~K}$ is assumed. This is the strongest assumption that can produce a ratio $0.2 \mathrm{dex}$ lower, if the temperature is $2000 \mathrm{~K}$ lower. Correspondingly, a 0.2 dex higher ratio would require the temperatures to be higher by $\sim 3000 \mathrm{~K}$. However, such a high electron temperature is unexpected since most regions are not particularly oxygen poor. A low temperature $(<8000 \mathrm{~K})$ would imply a N/O ratio lower than found for most metal poor galaxies $(\log (\mathrm{N} / \mathrm{O})=-1.8)$. The uncertainties in the line fluxes imply an uncertainty of $0.1 \mathrm{dex}$ in $\log (\mathrm{N} / \mathrm{O})$. Since the densities of the various regions are close to $100 \mathrm{~cm}^{-1}$, the small differences do not imply a significant correction to the $\mathrm{N} / \mathrm{O}$ ratio. Overall this implies an uncertainty in the $\mathrm{N} / \mathrm{O}$ ratio of $0.2 \mathrm{dex}$.

The N/O ratio can be used to probe the star formation history of the galaxy given the different timescales for the primary nitrogen production and the secondary contribution of nitrogen from intermediate mass stars. Compared to the solar value of $\log (\mathrm{N} / \mathrm{O})_{\odot}=-0.81$ (Holweger 2001), most of the regions have sub solar values. Within the uncertainties the WR and SN regions have similar values of $\mathrm{N} / \mathrm{O}$, while the value for the entire galaxy is again dominated by these bright $\mathrm{H}$ II regions. Taken at face value, the $\mathrm{N} / \mathrm{O}$ ratios indicate that the $\mathrm{WR}$ region is the youngest, but not as young as the suggested by the N/O ratio for some damped Lyman- $\alpha$ systems (Centurión et al. 2003; Pettini et al. 2008). Contamination from previous generations of stars to gas over timescales longer than 100-300 Myr in the WR region is responsible for this effect. The presence of WR stars is expected to increase the N/O ratio (Meynet \& Maeder 2005), although Izotov et al. (2006) found that the enhancement is small. For GRB hosts, it remains unclear whether an enhancement of nitrogen is present because the measurement errors are large (see Wiersema et al. 2007).

\subsection{Stellar population modelling}

The observed spectra were fit by the spectral templates derived from simple stellar evolution models from Starburst99 (Leitherer et al. 1999), which included both stellar and nebular emission. We chose to fit the VIMOS spectra with models of 0.4 solar metallicity because this is the most representative value for the host galaxy. The models assume an instantaneous burst of star formation, and a Salpeter law initial mass function between 1 and $100 M_{\odot}$. The best-fit templates are determined from a standard $\chi^{2}$ fitting technique. Using the intrinsic extinction in Table 2 to de-redden the spectra before fitting the models, we find that the best-fit age of $6 \mathrm{Myr}$ for the WR region, whereas the best-fit age is 3 Myr for the SN region due to the large intrinsic extinction. For all $\mathrm{H}$ II regions, the best-fit ages are between 2 and $10 \mathrm{Myr}$ as listed in Col. 4 in Table 3. With the smaller intrinsic reddening inferred from the SN region, Sollerman et al. (2005) found an age of $6 \mathrm{Myr}$, and a corresponding progenitor mass of $30 \pm 5 M_{\odot}$.

For comparison, we also fitted the de-reddened spectra from individual regions to the high resolution spectral templates from Bruzual \& Charlot (2003). The models assumed a single burst population and a Salpeter law initial mass function and again a 0.4 solar metallicity. We generally found a larger scatter between individual H II region with best fit ages between 1 and 200 Myr. Since these models did not include the nebular emission component, which is dominant for the very young regions, the ages determined from the Starburst99 model should represent the regions more appropriately. 
Table 3. Table for derived quantities for the different regions. The last row gives the mean and standard deviation for all the regions. Column 2 is the SFR derived from the observed $\mathrm{H} \alpha$ flux, and Col. 3 corrects the SFR for the extinction given in Table 2. Column 4 gives the best fit age. The mass (Col. 5) is determined from population modelling. The mass to light ratio (Col. 6) uses the luminosity $L_{B}$ derived from the $B$ magnitude relative to the $A B$ magnitude for the sun $M_{B, \odot}=5.33$. Column 7 lists the specific SFR per Gyr normalised to the mass after correction for the host extinction, and Col. 8 lists the luminosity specific SFR, both derived after correcting for intrinsic extinction.

\begin{tabular}{|c|c|c|c|c|c|c|c|}
\hline $\begin{array}{l}\text { Reg. } \\
\text { (1) }\end{array}$ & $\begin{array}{c}\text { SFR } \\
\left(\times 10^{-3} M_{\odot} \mathrm{yr}^{-1}\right) \\
(2)\end{array}$ & $\begin{array}{c}\text { SFR (corr.) } \\
\left(\times 10^{-3} M_{\odot} \mathrm{yr}^{-1}\right) \\
(3)\end{array}$ & $\begin{array}{r}\text { Age } \\
(\mathrm{Myr}) \\
(4)\end{array}$ & $\begin{array}{c}\text { Mass } \\
\left(\times 10^{6} M_{\odot}\right) \\
\quad(5)\end{array}$ & $\begin{array}{c}M / L_{B} \\
\left(M_{\odot} / L_{B, \odot}\right) \\
(6)\end{array}$ & $\begin{array}{c}\text { Mass SSFR (corr.) } \\
\left(M_{\odot} \mathrm{Gyr}^{-1} M_{\odot}^{-1}\right) \\
(7)\end{array}$ & $\begin{array}{c}\text { Lum SSFR (corr.) } \\
\left(M_{\odot} \mathrm{yr}^{-1}(L / L *)^{-1}\right) \\
(8)\end{array}$ \\
\hline Galaxy & 233.0 & 447.0 & 2 & 20.1 & 0.014 & 22.2 & 10.9 \\
\hline WR reg. & 78.3 & 113.3 & 2 & 0.44 & 0.007 & 257. & 58.2 \\
\hline SN reg. & 5.84 & 22.9 & 3 & 0.50 & 0.035 & 45.8 & 54.3 \\
\hline WR & 54.5 & 58.0 & 6 & 0.28 & 0.007 & 207. & 48.9 \\
\hline \multirow[t]{22}{*}{$\mathrm{SN}$} & 2.52 & 12.9 & 3 & 0.20 & 0.035 & 64.3 & 76.4 \\
\hline & 1.22 & 20.1 & 3 & 1.15 & 0.149 & 17.5 & 89.1 \\
\hline & 1.20 & 5.25 & 3 & 0.18 & 0.057 & 29.2 & 56.8 \\
\hline & 1.01 & 5.48 & 3 & 0.43 & 0.060 & 12.7 & 26.1 \\
\hline & 2.09 & 5.32 & 4 & 0.22 & 0.020 & 24.2 & 16.7 \\
\hline & 1.45 & 4.99 & 4 & 0.19 & 0.033 & 26.2 & 29.6 \\
\hline & 2.30 & 26.1 & 3 & 0.46 & 0.096 & 56.8 & 185.1 \\
\hline & 1.02 & 3.45 & 3 & 0.28 & 0.028 & 12.3 & 11.7 \\
\hline & 6.31 & 12.6 & 6 & 0.20 & 0.012 & 63.2 & 26.3 \\
\hline & 0.98 & 3.61 & 3 & 0.35 & 0.034 & 10.3 & 12.0 \\
\hline & 0.68 & 2.92 & 3 & 0.26 & 0.041 & 11.2 & 15.7 \\
\hline & 2.24 & 4.49 & 3 & 0.13 & 0.010 & 34.5 & 12.3 \\
\hline & 1.43 & 20.6 & 3 & 0.46 & 0.122 & 44.8 & 185.3 \\
\hline & 1.71 & 3.73 & 10 & 0.57 & 0.038 & 6.55 & 8.43 \\
\hline & 2.09 & 3.08 & 10 & 0.29 & 0.020 & 10.6 & 7.09 \\
\hline & 2.07 & 2.07 & 3 & 0.08 & 0.005 & 25.9 & 4.39 \\
\hline & 5.58 & 5.58 & 2 & 0.07 & 0.012 & 79.8 & 32.0 \\
\hline & 1.41 & 2.64 & 6 & 0.10 & 0.007 & 26.4 & 6.13 \\
\hline & 3.52 & 3.52 & 3 & 0.12 & 0.005 & 29.4 & 4.58 \\
\hline & 0.48 & 1.27 & 3 & 0.11 & 0.017 & 11.6 & 7.48 \\
\hline & 1.20 & 1.20 & 3 & 0.04 & 0.004 & 30.1 & 4.19 \\
\hline & 0.95 & 0.95 & 3 & 0.03 & 0.004 & 31.5 & 4.80 \\
\hline Mean & 4. \pm 11 & $9 . \pm 13$ & $4 \pm 2$ & $0.3 \pm 0.2$ & $0.04 \pm 0.04$ & $38 . \pm 42$ & $38 . \pm 52$ \\
\hline
\end{tabular}

The ages derived from the individual regions are in broad agreement with those derived from the equivalent widths in Sect. 3.4, which indicate ages of 3 to $6 \mathrm{Myr}$ for the individual HII regions, and the emission line ratios in Sect. 4. Discrepancies in the individual age estimates are probably caused by uncertainties in the reddening estimate, which affect the spectral fittings, while the $E W$ s are largely unaffected by the reddening. The continuum provides an insight into the older populations of stars, while the emission lines represent the most recent star forming episode. Since the younger regions have higher extinctions, this could affect the $E W \mathrm{~s}$. Irrespectively of this fact, both the $E W s$ and the population fittings indicate young ages for all regions ( $<10 \mathrm{Myr})$.

\subsection{Mass estimates}

The best-fit Starburst 99 models were used to estimate the masses of the different $\mathrm{H}$ II regions. Since the template models were created for stellar populations of $10^{6} M_{\odot}$, a simple scaling to the observed spectrum was required to derive the mass. Using the first entries in Table 2, the WR and $\mathrm{SN}$ regions have similar masses of $0.44 \times 10^{6} M_{\odot}$ and $0.5 \times 10^{6} M_{\odot}$, respectively, while the mass of the luminous stars in the galaxy is $2 \times 10^{7} M_{\odot}$. Uncertainties in the mass estimate includes both the error for the absolute flux, and the correction for the reddening yielding a total uncertainty of $20 \%$.
In Table 3, the masses are used with estimates of the SFR to derive additional parameters, such as mass-to-light ratios and specific SFRs, using mass as a means of normalization instead of the luminosity for the various regions. We note that the units in Cols. 7 and 8 are different. We checked the mass estimates for the individual regions by comparing the FORS $R$ band magnitudes, extracted within the same aperture as the VIMOS spectra, with the model predictions of the 0.4 solar metallicity Bruzual \& Charlot (2003) models. We found a generally good agreement with the values listed in Table 3. The derived mass depended strongly on the assumed age for the regions and the extinction applied.

Comparing the mass SSFR with the definition of the luminosity SSFR used in Sect. 3.6 (the two final columns in Table 3), the values agree in general to within a factor of a few. The determination of the "mass SSFR" involves additional uncertainties because of the model dependencies of the mass-to-light ratio and age determination. These uncertainties do not influence the luminosity SSFR since this quantity only includes directly observed parameters and is therefore an excellent proxy for the more generally used mass SSFR. The SSFRs in the table are higher compared to those derived for higher redshift GRB hosts, which have typical extinction-corrected luminosity SSFRs $=1-30 M_{\odot} \mathrm{yr}^{-1}$ $\left(L / L^{*}\right)^{-1}$ (Christensen et al. 2004a). The reason is that the extinction determined from the emission line ratios in the $\mathrm{H}$ II regions was far higher than found from the integrated spectral energy 
distributions, and the correction applied to the SFRs was therefore correspondingly higher.

For the entire galaxy, we compared the mass derived from the stellar population model fitting to masses derived from the absolute magnitudes measured from the FORS $R$ band image. Within the VIMOS FOV, the ratio of the luminosity is $L / L_{\odot}=$ $10^{9.3}$. Assuming a mass-to-light ratio of 0.1 (calibrated using the $K$ band as appropriate for GRB hosts cf. Savaglio et al. 2008), the mass within the field was $2 \times 10^{8} M_{\odot}$. In comparison, the mass estimated from the kinematical data was a factor of three times higher. We tested this mass estimate with the spectral energy distribution fitting codes Z-Peg (Le Borgne \& Rocca-Volmerange 2002), and HyperZ (Bolzonella et al. 2000) on the measured broad band magnitudes. Both SED fitting codes provide similar measurements of mass to within the uncertainties.

To investigate whether the galaxy is unusual in comparison with field galaxies, we compared our SFR measurement with SFRs measured for SDSS galaxies. The total stellar mass of the host galaxy that we study, infers an expected star formation rate of $0.3 M_{\odot} \mathrm{yr}^{-1}$ following the distribution values for galaxies in the SDSS (Brinchmann et al. 2004). The $\mathrm{H} \alpha$ flux corrected for the host extinction in Sect. 3.6 is $0.4 M_{\odot} \mathrm{yr}^{-1}$, which is within the distribution of SFRs for SDSS galaxies of similar mass. With this SFR and a total mass of $2 \times 10^{8} M_{\odot}$, the specific SFR for the host $\left(S S F R=2.0 M_{\odot} \mathrm{Gyr}^{-1} M_{\odot}{ }^{-1}\right)$ is located at the upper end of the distribution for SDSS galaxies (see Fig. 24 of Brinchmann et al. 2004).

\subsection{Variation of properties in the HII regions}

To illustrate the various average properties in Table 2 and 3 relative to the values measured for the SN and WR regions, we calculate for each quantity the difference from the average (written here for the SFR):

Deviation $=\frac{\mathrm{SFR}(\mathrm{SN})-\langle\mathrm{SFR}(\mathrm{H} \mathrm{II})\rangle}{\sigma(\mathrm{SFR}(\mathrm{H} \mathrm{II}))}$,

where $\langle\mathrm{SFR}(\mathrm{HII})\rangle$ is the mean value for the SFR, and $\sigma(\mathrm{SFR}(\mathrm{HII}))$ is the standard deviation as listed in the last row in Tables 2 and 3.

Figure 8 shows this quantity for all other properties. Notably the SFRs (corrected and uncorrected for the host extinction) of the WR region are significantly $(4 \sigma)$ above the average for all $\mathrm{H}$ II regions, and likewise the abundances are lower for both the WR and SN regions, although the values are consistent with the average within the uncertainties. Since the masses of the WR and $\mathrm{SN}$ regions are similar, it follows that the extinction-corrected mass SSFR is also much higher for the WR region. On the other hand, the luminosity SSFRs for both the SN and WR regions appear to be only slightly above the average. Since the $B$ band magnitude of the $\mathrm{SN}$ region is much fainter than for the WR region, and no correction for extinction is applied to the magnitude before the luminosity SSFRs are calculated, the luminosity weighted SSFR for the two regions are similar and close to the average for the HII regions. For none of the parameters does the figure change significantly if the median value is used in the calculation instead of the average.

The difference compared to the values in the SSFR map in Fig. 6 is due to the fact that the map is uncorrected for internal extinction in the host, whereas the SSFRs in the tables and in Fig. 8 are extinction corrected.

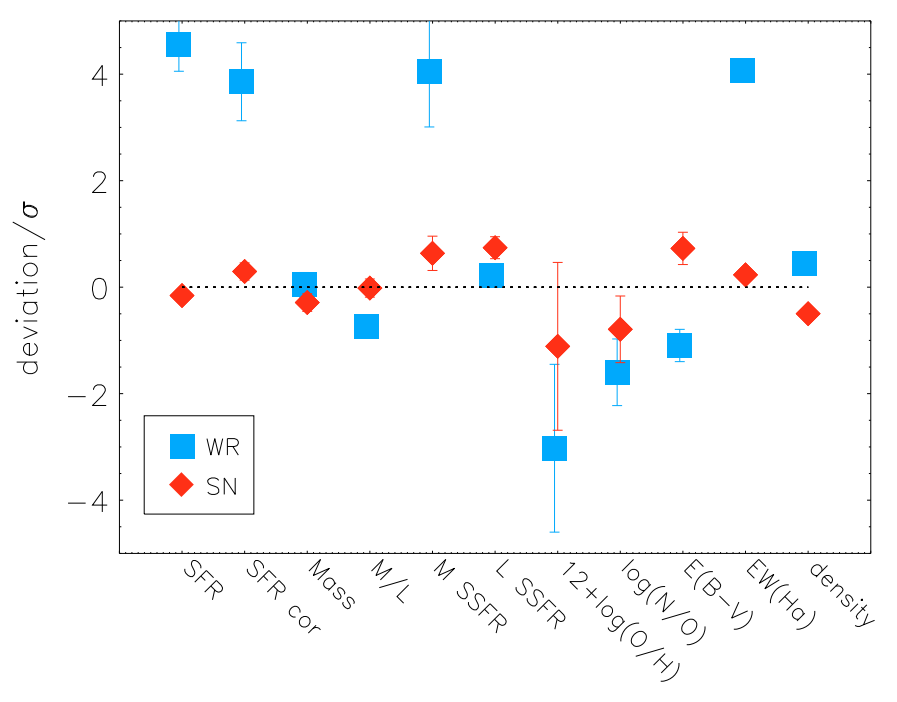

Fig. 8. Deviation from the average of the properties listed on the $x$-axis. The WR region is indicated by squares and the $\mathrm{SN}$ region by diamonds. Error bars are in some cases smaller than the symbols.

\section{Emission line ratios, ages, and metallicities}

While the $\mathrm{SN}$ region in the GRB 980425 host is the second most metal-poor region, it is not dust-free and most properties (SFRs, $E W \mathrm{~s}$, masses) are similar to those of the remaining $\mathrm{H}$ II regions in the host galaxy. In contrast, the WR region appears to be characteristic of that expected for a GRB region in terms of these properties (see Christensen et al. 2004a).

We compare the emission line ratios $[\mathrm{O}$ III $] / \mathrm{H} \beta$ vs. $[\mathrm{N} \mathrm{II}] / \mathrm{H} \alpha$ and $[\mathrm{S} \mathrm{II}] / \mathrm{H} \alpha$ to other GRB hosts and other star-forming galaxies in Fig. 9. The individual regions (uncorrected for the internal extinction in the host) from Table 2 are represented by the small squares. De-reddening the fluxes should not affect the line ratios significantly. The emission line ratios are compared with those of other GRB host found in the literature. The emission line ratios were usually obtained from integrated spectra of the hosts.

Figure 9 also includes the theoretical emission line ratios for models of H II region of various metallicities and ages (Dopita et al. 2006). Instead of the frequently used ionization parameter, the models involve a parameter, $R$, which represents the fraction of the star cluster mass and the pressure in the interstellar medium. For typical stellar masses of the $\mathrm{H}$ II regions between $10^{5}-10^{6} M_{\odot}$ in Table 3 , and a temperature of $10^{4} \mathrm{~K}$ and a density of $100 \mathrm{~cm}^{-3}$ in Sect. 3.7, this implies that $-1<\log R<0$. The tracks in Fig. 9 assume the fraction $\log R=0$. For different choices of $R$, the model ages of the H II regions differ slightly (for details see Dopita et al. 2006), but this will not change the conclusion for the relative ages of the GRB host emission line regions.

Except for GRB 060505 and GRB 060614, all GRB hosts are located at or above the $0.1 \mathrm{Myr}$ model, with their model metallicities between 0.4 and 1 solar. The offset between the models and the data points in the right hand panel, which use the [S II] $/ \mathrm{H} \alpha$ ratio, is real, and probably due to a deficiency of the models because they do not include emission from a diffuse radiation field, which would increase the [S II] flux. Furthermore, the models of different metallicities for [S II] provide prediction that are close to each other, whereas they are distinctly separated in the $[\mathrm{N} \mathrm{II}]$ panel. Hence, the $[\mathrm{N} \mathrm{II}] / \mathrm{H} \alpha$ line ratio is a more effective tool for discriminating between metallicities than the $[\mathrm{S} \mathrm{II}] / \mathrm{H} \alpha$ line ratio. This may explain why the data for the 

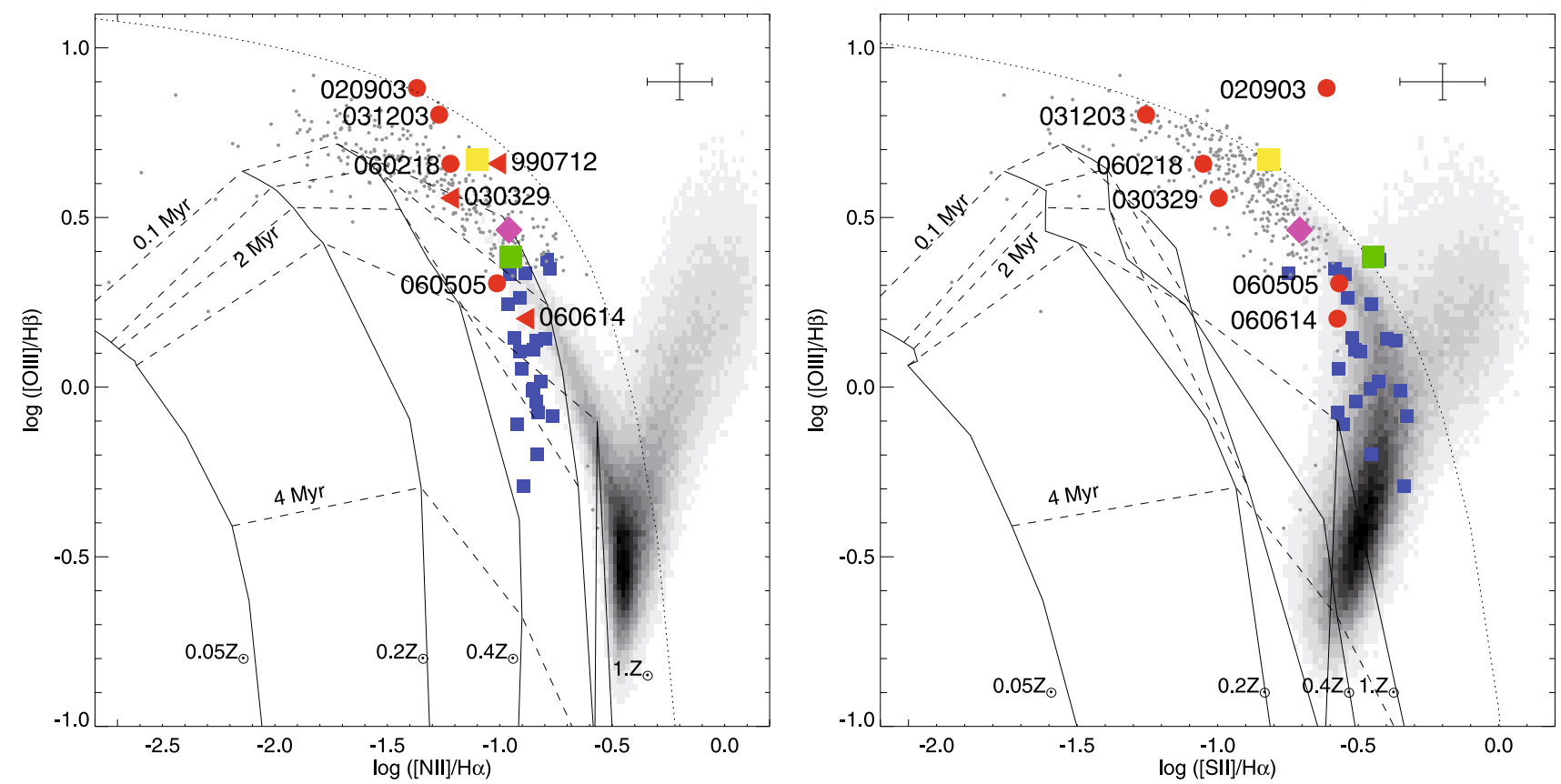

Fig. 9. Emission line ratios for GRB hosts: $[\mathrm{O} \mathrm{III}] / \mathrm{H} \beta$ vs. $[\mathrm{N} \mathrm{II}] / \mathrm{H} \alpha$ (on the left) and vs. [S II]/H $\alpha$ (on the right). Individually resolved $\mathrm{H}$ II regions are shown as small blue squares, the SN region (larger green square), and the WR region (large yellow square), and the entire galaxy (pink diamond). The median error bar for the sample is indicated in the upper right corner. The distinct location of the WR and SN regions indicate that they are special relative to other $\mathrm{H}$ II regions. The evolutionary models that link emission line regions at ages from 0.1 to 5 Myr for 0.05 , $0.2,0.4$ and solar metallicities are taken from Dopita et al. (2006). The dotted, curved lines denote the separation of pure star forming galaxies to AGN dominated emission line ratios (Kewley et al. 2001; Kauffmann et al. 2003). To compare with field galaxies, the gray scale area represent SDSS galaxies (Tremonti et al. 2004), and the small grey dots SDSS galaxies with metallicities between 0.03 and 0.7 solar (Izotov et al. 2006). Emission line ratios for other GRB hosts are overlayed by the named red circles. Two hosts have upper detection limits for [N II] and are represented by red triangles (right hand limits); GRB 030329 (Gorosabel et al. 2005) and GRB 990712 (Christensen et al. 2004b). Other references: GRB990712: Küpcï Yoldaş et al. (2006), GRB 031203: Prochaska et al. (2004), GRB 020903: Hammer et al. (2006), GRB 060218: Wiersema et al. (2007), GRB 060614: Gal-Yam et al. (2006), GRB 060505: Thöne et al. (2008). A database is available at the GHostS web site (www. grbhosts.org). See the electronic edition of the Journal for a colour version of this figure.
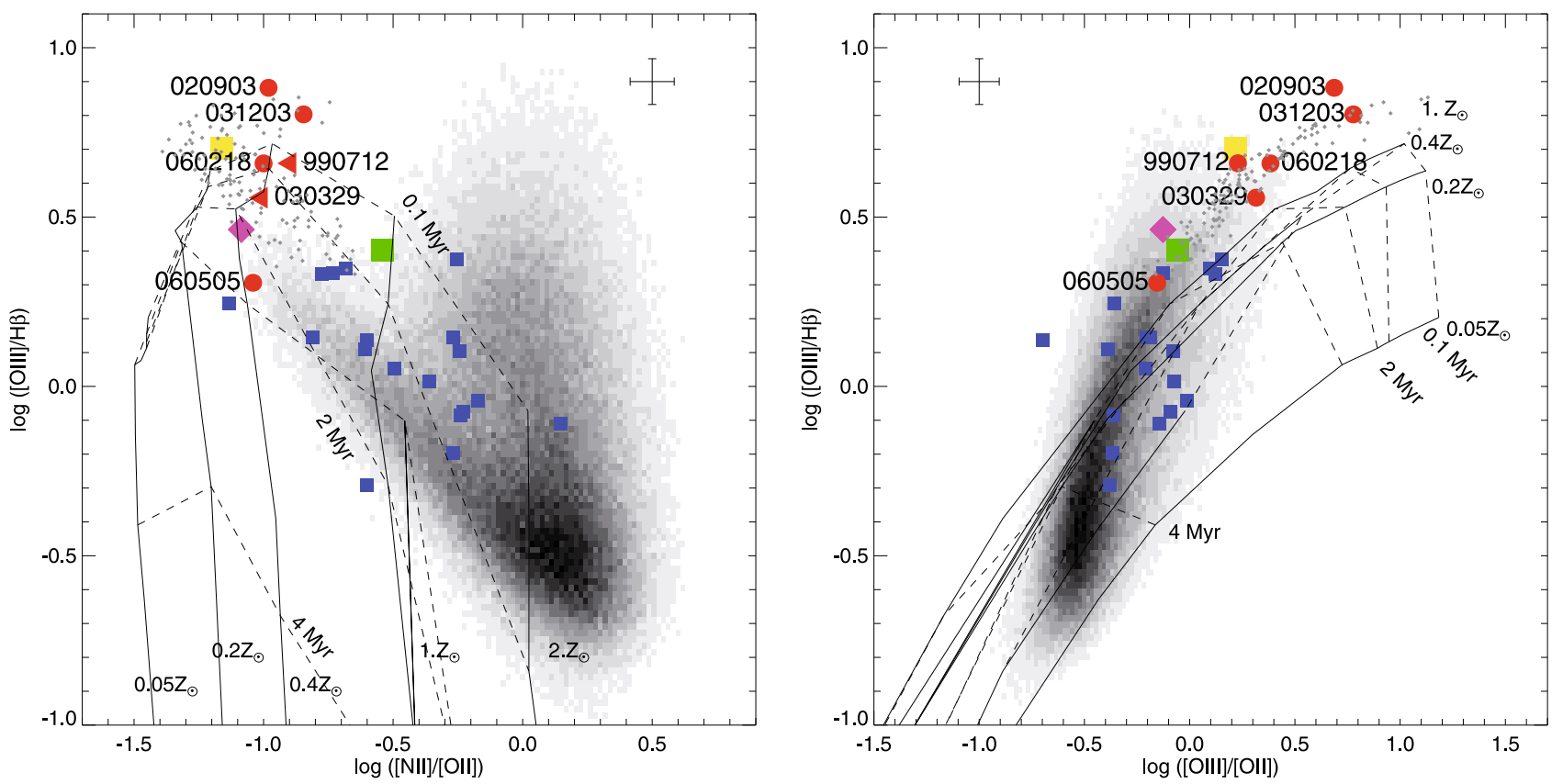

Fig. 10. Emission line ratios $[\mathrm{O}$ III $] / \mathrm{H} \beta$ vs. [N II $] /[\mathrm{O}$ II] (on the left) and vs. [O III]/[O II] (on the right). The symbol shapes and colours are the same as in Fig. 9. 
$\mathrm{H}$ II regions are clearly separate from the SDSS galaxies in the left panel, because they are more metal poor than the SDSS galaxies, while their data coincide in the right hand panel. The important point is that in this plot the GRB regions are offset towards the higher $[\mathrm{O} \mathrm{III}] / \mathrm{H} \beta$ ratio values (smaller age) but still have a range of metallicities. Figure 10 presents a similar plot for the line ratios: $[\mathrm{O} \mathrm{III}] / \mathrm{H} \beta$ vs. $[\mathrm{N}$ II $] /[\mathrm{O}$ II $]$ and $[\mathrm{O}$ III $] /[\mathrm{O}$ II $]$. We note that, due to the relatively large uncertainties in the [O II] line fluxes, the scatter is larger, but trends similar to those in Fig. 9 are evident.

It is interesting to note that in both panels of Figs. 9 and 10, the WR data occupy a similar region of the plot as most of the other GRB hosts, which are significantly offset towards the top. Similarly the SN region is located towards the top relative to the other H II regions in the host. This would indicate that GRBs occur in significantly younger regions - at least in the low redshift hosts where the emission line ratios can be studied.

The GRB 060505 host was also studied by resolved spectroscopy i.e. by long slit observations (Thöne et al. 2008). The symbols in Figs. 9, and 10 correspond to the emission line ratios for the region where the GRB occurred. Emission lines from four other regions are analysed, and although we do not show the individual regions for this host here, the GRB region is also offset to the top of the figure relative to the other regions. In the GRB 060505 host, this implies that the GRB occurred in the youngest region probed by the long slit spectrum.

We also compare the emission line ratios with the integrated ones for field galaxies in the SDSS. The gray scale background images represent more than 500000 galaxies in the SDSS DR $4{ }^{4}$ (Tremonti et al. 2004). SDSS galaxy spectra that have signatures of WR stars span the full range of emission line ratios measured for other SDSS galaxies (Brinchmann et al. 2008). Clearly not all WR regions in field galaxies are potential progenitor regions for GRBs. Most of these SDSS galaxies are relatively bright, massive, and hence metal rich; a more appropriate comparison sample might therefore be a metal poor sample of galaxies. In Figs. 9 and 10, the small individual dots represent galaxies with metallicities between 0.03 and 0.7 solar from the SDSS (Izotov et al. 2006). The selection criteria for the various samples were completely different: the SDSS galaxies were a flux-limited sample, the metal poor galaxies were a small sub-sample of the SDSS galaxies with a clear detection of the [O III] $\lambda 4363$ line, while the GRB hosts were not selected by either of these selection criteria. The GRB hosts occupy similar regions of the diagram as the metal poor SDSS galaxies, according to the models, they do not occupy the very metal poor region of the plots.

Most of the data for the individual H II regions in the GRB 980425 host occupy a slightly sub solar metallicity region compared to the SDSS galaxies. The scatter in the positions is real and reflects both the age and metallicity spread from one region to the next. As determined in Sect. 3.8, the host galaxy has an oxygen abundance of $0.3-0.8$ solar, which is consistent with the model curves in the left panel, where the small squares occupy the model space between these two metallicities.

We can compare these model ages for the individual regions in the GRB 980425 host with those determined from the $\mathrm{H} \alpha$ and $\mathrm{H} \beta E W$ measurements in Sect. 3.4. The WR region has an age of 3 Myr according to the $E W$, while younger ages are suggested by the models in Fig. 9. This is surprising since a very young region $(<1 \mathrm{Myr})$ is expected to have a high intrinsic extinction, however the IR emission seen in the Spitzer images (Le Floc'h et al. 2006) does not indicate an optically hidden population of

${ }^{4}$ http://www.mpa-garching.mpg.de/SDSS/ young stars that is not inferred from the $\mathrm{H} \alpha$ emission. The observations of WR features imply that stellar populations with ages around 3 Myr must be present. The $\mathrm{SN}$ region and the integrated spectrum of the galaxy lie between the 1 and 2 Myr evolutionary model line in the plot, i.e. similar to the ages determined from the population model fits, and comparable to the ages derived from the $E W \mathrm{~s}$.

\section{Discussion and conclusions}

\subsection{Implication for high-z GRB hosts}

In this paper, we have studied the properties of resolved regions in the very nearby host galaxy of GRB 980425/SN 1998bw. We found that the HII region where the GRB/SN occurred has the second lowest oxygen abundance, while the lowest value arises in the WR region. Apart from the metallicity, other physical properties in the $\mathrm{SN}$ region are representative of the integrated spectrum of the host galaxy.

The variations, especially those of the metallicity and extinction, over the face of the galaxy have important implications for the interpretation of the observations of higher redshift GRB hosts. In the case that $z>1$ GRB hosts do not contain just one (giant) star forming region or star cluster, some properties will not be recoverable when the only information available is the integrated properties of the entire galaxy. In several GRB hosts, the environment appears to be complex with a few or several individual star-forming regions that can only be resolved at the spatial resolution of the HST (e.g. Hjorth et al. 2002).

Based on the study of the resolved population of the GRB 980425 host, we identified the following effects that would occur if the host was at higher redshift and unresolved:

- The integrated spectra from the separate regions would decrease the emission line equivalent widths corresponding to individual regions, because older stellar populations would be covered. This would then substantially decrease the inferred age of unresolved star burst regions.

- The specific star formation rates would be much higher in individual H II regions than the integrated one where less active regions are also covered.

- For the GRB 1998bw host galaxy, the scatter in oxygen abundances between different regions would be relatively small compared to the overall uncertainty in the calibrations. Abundances would be similar to within the $3 \sigma$ uncertainties. For high redshift GRB hosts, the integrated spectra may produce a fairly good representation of the abundances in the GRB regions.

- We found that the luminosity SSFR introduced in Christensen et al. (2004a) for GRB hosts is a good proxy for the mass SSFR, which is more frequently used in the literature. Furthermore, the integrated SSFR of the GRB 980425 host is similar to other GRB hosts.

- The extinction for small isolated regions can be severely underestimated. The overall host has a low reddening of $E_{B-V}=0.3 \mathrm{mag}$, which is normal for (unresolved) higher redshift GRB hosts. The $\mathrm{SN}$ region itself has a higher than average value. Relative to spectral energy distribution fitting of GRB host broad band data, the emission line ratios indicated a higher extinction.

- The emission line ratios for the different regions in the host indicated that most other GRB hosts (at $z<0.4$ ) have properties that would seem similar to the WR region in the GRB 980425 host. This implies that even though we cannot 
resolve spatially some GRB hosts, they must be dominated by very young stars, even though we cannot always distinguish any WR features in their spectra.

- The separation of $800 \mathrm{pc}$ between the WR and SN region corresponds at $z=1$ to a single pixel with the HST spatial resolution of $0{ }^{\prime} 1$. Had the host been at $z=1$, we would have been able to resolve only two distinct regions: the centre of the galaxy and the WR region. In a broad band HST image, the GRB location would coincide with the brightest pixel as seen for many cosmological GRBs (Fruchter et al. 2006).

\subsection{GRB host metallicities}

If low metallicities are a necessary prerequisite to creating GRBs, this effect should be immediately apparent from the properties of the host galaxies. Theoretical models suggest that GRBs correspond to star forming galaxies with lower than $10 \%$ solar metallicity (Yoon et al. 2006). Models for winds from WR stars are based on Fe as drivers (Vink \& de Koter 2005), and likewise the GRB progenitor models are based on the Fe abundance (Yoon \& Langer 2005) where no GRB progenitors are expected from stars with metallicities larger than $0.1-0.3$ solar (Woosley \& Heger 2006; Langer \& Norman 2006). However, while the models are based on the Fe metallicity, the emission lines from the host galaxies trace the oxygen abundance.

As demonstrated in Figs. 9 and 10, regions with higher metallicities than the model predictions are generally found among GRB hosts, although these are still below the solar value (see also Savaglio et al. 2008). A potential bias comes from the fact that the hosts are generally very faint, so the metallicity determination has to rely on strong emission line diagnostics, which have large uncertainties. The use of strong emission lines to derive abundances can in some cases infer a higher metallicity estimate than direct measurement from the [O III] $\lambda 4363$ or the [N II] $\lambda 5755$ emission lines. In the WR region, the strong line diagnostics infer a 0.3 solar oxygen abundance, while the direct measurement indicates a near solar value. In contrast, the O3N2 diagnostics for the GRB 060218 host indicates a 30\% solar oxygen abundance, while the direct method gives just $8 \%$ solar (Wiersema et al. 2007).

GRB hosts at $z>2$ have metallicities of about $10 \%$ solar in general as estimated from absorption lines in the afterglows (Savaglio 2006). However, as shown by Vreeswijk et al. (2007) for the case of GRB 060418, the rest frame UV absorption lines do not arise in the immediate GRB progenitor environment, but instead the interstellar medium of the host galaxy along one single line-of-sight. On the other hand, as we have discussed in this paper, the GRB hosts at low redshift are not necessarily very metal poor. The question is how to relate the low and high redshift sample of GRB hosts to each other when the observing techniques are completely different. Different biases are included from the assumption that the metallicity of the progenitor can be constrained from absorption lines in the GRB afterglow, and that emission lines from the host galaxy can infer the abundance at the progenitor site. Any of these metallicities may not be representative of the GRB progenitor itself. To understand how these two methods compare with each other, it is necessary to detect the emission lines and derive abundances from GRB hosts at $z>2$ in a similar way as performed routinely for local galaxies, as shown in this paper. By comparing the absorption metallicity, determined from the GRB afterglow, with abundances derived from emission lines it can be verified if the metallicity is representative of the host as a whole, and to place the GRB hosts in relation to star-forming field galaxies at high and low redshifts. The comparison of absorption lines and emission line spectroscopy for a high redshift GRB host galaxy can provide a measure of the $\mathrm{Fe} / \mathrm{O}$ ratio. Since the GRB hosts are young and show intense SSFRs, it is likely that the oxygen abundance is higher than that derived from $\mathrm{Fe}$ due to the relatively small contribution from $\mathrm{SN}$ type Ia. Hence, the determination of the $\mathrm{Fe} / \mathrm{O}$ ratio can provide constraints on the star formation history in galaxies fainter than those generally studied in flux-limited surveys.

Acknowledgements. L.C. acknowledges the hospitality of the people at the Dark Cosmology Center in Copenhagen, where much of the data analysis was done. The Dark Cosmology Centre is funded by the Danish National Research Foundation. P.M.V. acknowledges the support of the EU under a Marie Curie Intra-European Fellowship, contract MEIF-CT-2006-041363. J.S. is a Royal Swedish Academy of Sciences Research Fellow supported by a grant from the Knut and Alice Wallenberg Foundation. Support for ELF's work was provided by NASA through the Spitzer Space Telescope Fellowship Program. This research has made use of the GHostS database (http://www.grbhosts.org), which is partly funded by Spitzer/NASA grant RSA Agreement No. 1287913. We thank Göran Östlin for the $\mathrm{H} \alpha$ data check, Sandra Savaglio for useful suggestions and discussions, and Damien Le Borgne for sharing the Z-Peg code, and finally Avishay Gal-Yam for sharing the Gemini spectrum of the GRB 060614 host. We thank the referee for a constructive report.

\section{References}

Asplund, M., Grevesse, N., Sauval, A. J., Allende Prieto, C., \& Kiselman, D. 2004, A\&A, 417, 751

Becker, T. 2002, Ph.D. Thesis, Astrophysikalisches Institut Potsdam, Germany Bessell, M. S. 1990, PASP, 102, 1181

Bloom, J. S., Kulkarni, S. R., \& Djorgovski, S. G. 2002, AJ, 123, 111

Bolzonella, M., Miralles, J.-M., \& Pelló, R. 2000, A\&A, 363, 476

Brinchmann, J., Charlot, S., White, S. D. M., et al. 2004, MNRAS, 351, 1151

Brinchmann, J., Kunth, D., \& Durret, F. 2008, A\&A, 485, 657

Bruzual, A. G., \& Charlot, S. 2003, MNRAS, 344, 1000

Castro Cerón, J. M., Michalowski, M. J., Hjorth, J., et al. 2008, [arXiv:0803.2235]

Centurión, M., Molaro, P., Vladilo, G., et al. 2003, A\&A, 403, 55

Chen, H.-W., Prochaska, J. X., Bloom, J. S., \& Thompson, I. B. 2005, ApJ, 634, L25

Chen, H.-W., Prochaska, J. X., Ramirez-Ruiz, E., et al. 2007, ApJ, 663, 420 Christensen, L., Hjorth, J., \& Gorosabel, J. 2004a, A\&A, 425, 913

Christensen, L., Hjorth, J., Gorosabel, J., et al. 2004b, A\&A, 413, 121

Dessauges-Zavadsky, M., Chen, H.-W., Prochaska, J. X., Bloom, J. S., \& Barth, A. J. 2006, ApJ, 648, L89

Dopita, M. A., Fischera, J., Sutherland, R. S., et al. 2006, ApJS, 167, 177 Filippenko, A. V. 1982, PASP, 94, 715

Fitzpatrick, E. L. 1999, PASP, 111, 63

Fruchter, A. S., Levan, A. J., Strolger, L., et al. 2006, Nature, 441, 463 Fynbo, J. P. U., Holland, S., Andersen, M. I., et al. 2000, ApJ, 542, L89 Gal-Yam, A., Fox, D. B., Price, P. A., et al. 2006, Nature, 444, 1053 Galama, T. J., Vreeswijk, P. M., van Paradijs, J., et al. 1998, Nature, 395, 670 Gehrels, N., Chincarini, G., Giommi, P., et al. 2004, ApJ, 611, 1005

González Delgado, R. M., Leitherer, C., \& Heckman, T. M. 1999, ApJS, 125, 489

Gorosabel, J., Pérez-Ramírez, D., Sollerman, J., et al. 2005, A\&A, 444, 711

Hammer, F., Flores, H., Schaerer, D., et al. 2006, A\&A, 454, 103 Hjorth, J., Thomsen, B., Nielsen, S. R., et al. 2002, ApJ, 576, 113 Hjorth, J., Sollerman, J., Møller, P., et al. 2003, Nature, 423, 847

Holweger, H. 2001, in Joint SOHO/ACE workshop Solar and Galactic Composition, ed. R. F. Wimmer-Schweingruber, AIP Conf. Ser., 598, 23

Izotov, Y. I., Stasińska, G., Meynet, G., Guseva, N. G., \& Thuan, T. X. 2006, A\&A, 448, 955

Jakobsson, P., Levan, A., Fynbo, J. P. U., et al. 2006, A\&A, 447, 897

Kaneko, Y., Ramirez-Ruiz, E., Granot, J., et al. 2007, ApJ, 654, 385

Kauffmann, G., Heckman, T. M., Tremonti, C., et al. 2003, MNRAS, 346, 1055 Kennicutt, R. C. 1998, ARA\&A, 36, 189

Kennicutt, Jr., R. C., Calzetti, D., Walter, F., et al. 2007, ApJ, 671, 333

Kewley, L. J., \& Ellison, S. L. 2008, ApJ, 681, 1183

Kewley, L. J., Dopita, M. A., Sutherland, R. S., Heisler, C. A., \& Trevena, J. 2001, ApJ, 556, 121

Küpcü Yoldaş, A., Greiner, J., \& Perna, R. 2006, A\&A, 457, 115

Langer, N., \& Norman, C. A. 2006, ApJ, 638, L63

Le Borgne, D., \& Rocca-Volmerange, B. 2002, A\&A, 386, 446 
Le Floc'h, E., Duc, P.-A., Mirabel, I. F., et al. 2003, A\&A, 400, 499 Le Floc'h, E., Charmandaris, V., Forrest, W. J., et al. 2006, ApJ, 642, 636 Leitherer, C., Schaerer, D., Goldader, J. D., et al. 1999, ApJS, 123, 3

Malesani, D., Tagliaferri, G., Chincarini, G., et al. 2004, ApJ, 609, L5 Margutti, R., Chincarini, G., Covino, S., et al. 2007, A\&A, 474, 815 Meynet, G., \& Maeder, A. 2005, A\&A, 429, 581

Modjaz, M., Kewley, L., Kirshner, R. P., et al. 2008, AJ, 135, 1136 Monreal-Ibero, A., Roth, M. M., Schönberner, D., Steffen, M., \& Böhm, P. 2006, New Astron. Rev., 50, 426

Osterbrock, D. E. 1989, Astrophysics of gaseous nebulae and active galactic nuclei (University Science Books), 422

Pettini, M., \& Pagel, B. E. J. 2004, MNRAS, 348, L59

Pettini, M., Zych, B. J., Steidel, C. C., \& Chaffee, F. H. 2008, MNRAS, 385, 2011

Prochaska, J. X., Bloom, J. S., Chen, H.-W., et al. 2004, ApJ, 611, 200

Prochaska, J. X., Chen, H.-W., \& Bloom, J. S. 2006, ApJ, 648, 95

Prochaska, J. X., Chen, H.-W., Dessauges-Zavadsky, M., \& Bloom, J. S. 2007, ApJ, 666, 267

Savaglio, S. 2006, New J. Phys., 8, 195

Savaglio, S., Fall, S. M., \& Fiore, F. 2003, ApJ, 585, 638
Savaglio, S., Glazebrook, K., \& Le Borgne, D. 2008, [arXiv: 0803.2718]

Schlegel, D. J., Finkbeiner, D. P., \& Davis, M. 1998, ApJ, 500, 525

Shaw, R. A., \& Dufour, R. J. 1995, PASP, 107, 896

Sollerman, J., Holland, S. T., Challis, P., et al. 2002, A\&A, 386, 944

Sollerman, J., Östlin, G., Fynbo, J. P. U., et al. 2005, New Astron., 11, 103

Stanek, K. Z., Matheson, T., Garnavich, P. M., et al. 2003, ApJ, 591, L17

Starling, R. L. C., Wijers, R. A. M. J., Hughes, M. A., et al. 2005, MNRAS, 360, 305

Thöne, C. C., Fynbo, J. P. U., Östlin, G., et al. 2008, ApJ, 676, 1151 Tremonti, C. A., Heckman, T. M., Kauffmann, G., et al. 2004, ApJ, 613, 898 Vink, J. S., \& de Koter, A. 2005, A\&A, 442, 587

Vreeswijk, P. M., Ellison, S. L., Ledoux, C., et al. 2004, A\&A, 419, 927

Vreeswijk, P. M., Ledoux, C., Smette, A., et al. 2007, A\&A, 468, 83

Wiersema, K., Savaglio, S., Vreeswijk, P. M., et al. 2007, A\&A, 464, 529

Woosley, S. E. 1993, ApJ, 405, 273

Woosley, S. E., \& Heger, A. 2006, ApJ, 637, 914

Yin, S. Y., Liang, Y. C., Hammer, F., et al. 2007, A\&A, 462, 535

Yoon, S.-C., \& Langer, N. 2005, A\&A, 443, 643

Yoon, S.-C., Langer, N., \& Norman, C. 2006, A\&A, 460, 199 